\title{
The weighted Weiss conjecture and reproducing kernel theses for generalized Hankel operators
}

\author{
B. JACOB • E. RYDHE • A. WYNN
}

8th October 2013

Abstract The weighted Weiss conjecture states that the system theoretic property of weighted admissibility can be characterised by a resolvent growth condition. For positive weights, it is known that the conjecture is true if the system is governed by a normal operator; however, the conjecture fails if the system operator is the unilateral shift on the Hardy space $H^{2}(\mathbb{D})$ (discrete time) or the right-shift semigroup on $L^{2}\left(\mathbb{R}_{+}\right)$(continuous time). To contrast and complement these counterexamples, in this paper positive results are presented characterising weighted admissibility of linear systems governed by shift operators and shift semigroups. These results are shown to be equivalent to the question of whether certain generalized Hankel operators satisfy a reproducing kernel thesis.

Keywords One parameter semigroups, admissibility, Hardy space, weighted Bergman space, Hankel operators, Reproducing kernel thesis.

Mathematics Subject Classification (2000) 30H10, 30H20, 47B32, 47B35, 47D06, 93B28.

\section{Introduction}

Consider an infinite dimensional control system

$$
\begin{aligned}
& \dot{x}(t)=A x(t), \quad y(t)=C x(t), \quad t \geq 0, \\
& x(0)=x_{0} \in X
\end{aligned}
$$

where $A$ is the generator of a $C_{0}$-semigroup $(T(t))_{t \geq 0}$ on a Hilbert space $X$ and the observation operator satisfies $C \in \mathcal{L}(D(A), \mathbb{C})$. For the system to be well-posed, in the sense of [20], a necessary condition is that $C$ is admissible for $A$, that is, there exists $k>0$ such that

$$
\left\|C T(\cdot) x_{0}\right\|_{L^{2}\left(\mathbb{R}_{+}\right)} \leq k\left\|x_{0}\right\|_{X}, \quad x_{0} \in D(A) .
$$

An important consequence of admissibility is that the output $y$ can be well defined even in the case that $C$ is unbounded. In particular, admissibility implies that the map $x_{0} \mapsto C T(\cdot) x_{0} \in L^{2}\left(\mathbb{R}_{+}\right)$,

Fachbereich C - Mathematik und Naturwissenschaften, Bergische Universität Wuppertal, Gaußstraße 20, 42097 Wuppertal, Germany · Matematikcentrum Lunds Universitet, 22100 Lund, Sweden · Department of Aeronautics, Imperial College London, London, SW7 2AZ, United Kingdom, Tel. +44 (0)20 7594 5047, E-mail: a.wynn@imperial.ac.uk 
defined initially on $D(A)$, has a continuous extension to the whole space $X$, meaning that the output is well defined for any initial condition $x_{0} \in X$.

A generalization of admissibility, first considered in [4], is to require that the output is an element of a weighted $L^{2}$-space. For $\beta>-1, C$ is said to be $\beta$-admissible for $A$ if there exists a constant $k>0$ such that

$$
\int_{0}^{\infty} t^{\beta}\left|C T(t) x_{0}\right|^{2} d t \leq k^{2}\left\|x_{0}\right\|^{2}, \quad x_{0} \in D(A)
$$

To test whether a given system is $\beta$-admissible, a frequency-domain characterization is convenient and, to this end, it is not difficult to show that $\beta$-admissibility implies the resolvent growth condition

$$
\sup _{\lambda \in \mathbb{C}_{+}}(\operatorname{Re} \lambda)^{\frac{1+\beta}{2}}\left\|C R(\lambda, A)^{1+\beta}\right\|_{X^{*}}<\infty
$$

where $R(\lambda, A):=(\lambda I-A)^{-1}$ denotes the resolvent of the semigroup generator $A$, and $\mathbb{C}_{+}:=\{\lambda \in$ $\mathbb{C}: \operatorname{Re} \lambda>0\}$ is the right-half plane. The question of whether the converse statement $(2) \Rightarrow(1)$ holds, commonly referred to as a (weighted) Weiss conjecture, is much more subtle. Existing results concerning the conjecture are discussed below, but we first describe a discrete time version of the Weiss conjecture, introduced in [6], which will also be studied in this paper.

A discrete-time linear control system on a Hilbert space $X$ has the form

$$
x_{n+1}=T x_{n}, \quad y_{n}=C x_{n}, \quad x_{0} \in X, \quad n \in \mathbb{N},
$$

where $T \in \mathcal{L}(X)$ and $C \in X^{*}$. In this case, for $\beta>-1$, the observation functional $C$ is said to be (discrete) $\beta$-admissible for $T$ if there exists $k>0$ such that

$$
\sum_{n=0}^{\infty}(1+n)^{\beta}\left|C T^{n} x\right|^{2} \leq k^{2}\|x\|_{X}^{2}, \quad x \in X .
$$

Analogous to continuous time systems, the resolvent condition

$$
\sup _{\omega \in \mathbb{D}}\left(1-|\omega|^{2}\right)^{\frac{1+\beta}{2}}\left\|C(I-\bar{\omega} T)^{-(1+\beta)}\right\|_{X^{*}}<\infty
$$

is necessary for (3) and the discrete time form of the weighted Weiss conjecture is to ask when the converse implication is true. The Weiss conjecture is superficially easier to study in discrete time due to the boundedness of the operators involved. However, it should be noted that it is sometimes possible to translate positive and negative results concerning the conjecture via the Cayley transform $[6,21]$

The continuous time conjecture $(2) \Rightarrow(1)$ was originally posed [18] in the unweighted case $\beta=0$. In this situation, the conjecture is true if $A$ generates a $C_{0}$-semigroup of contractions [7], which extends the results that the conjecture holds if $A$ is normal [19] and if $A$ is the generator of the right-shift semigroup on $L^{2}\left(\mathbb{R}_{+}\right)[12]$. The discrete time version $(4) \Rightarrow(3)$ for $\beta=0$ and $T$ a contraction was shown in [6].

For $\beta \neq 0$, the behaviour of the conjecture is more complicated. In the case that $A$ is normal, the continuous time conjecture $(2) \Rightarrow(1)$ is true [23] for positive weight exponents $\beta \in(0,1)$, but false $[22]$ in the case that $\beta \in(-1,0)$. Analogous results also hold for the discrete time conjecture conjecture when $T$ is normal $[22,23]$. Furthermore, both continuous and discrete time conjectures are not true for general contraction operators when $\beta \in(0,1)$ : in continuous time, the right-shift semigroup on $L^{2}\left(\mathbb{R}_{+}\right)$provides the counterexample [21]; while in discrete time $(4) \Rightarrow(3)$ fails if $T$ is the unilateral shift on the Hardy space $H^{2}(\mathbb{D})[22]$.

It should be noted that the restriction $\beta \in(-1,1)$ in the above discussion arises from the fact that the growth bound $\sup _{\lambda \in \mathbb{C}_{+}}(\operatorname{Re} \lambda)^{\frac{1-\beta}{2}}\|C R(\lambda, A)\|<\infty\left(\right.$ respectively, the condition $\sup _{\omega \in \mathbb{D}}(1-$ 
$\left.|\omega|^{2}\right)^{\frac{1-\beta}{2}}\left\|C(I-\bar{\omega} T)^{-1}\right\|<\infty$ in discrete time) was considered in the cited literature, i.e. a condition involving only the first power of the resolvent. In this situation, the restriction $\beta<1$ is natural. However, as shown for example in [21], the truth of the weighted conjecture is not affected by considering instead the resolvent growth bound (2) and in this situation the natural range of weights is $\beta>-1$. Thus, the resolvent condition (2) is considered in the remainder of this paper.

The importance of determining the truth of the conjecture for the right-shift semigroup (or, in discrete time, the unilateral shift) is due to the Sz.Nagy-Foiaş model theory for contractions [15]. This states that a general contraction operator can be decomposed as a sum of operators, one of which is unitarily equivalent to a part of a shift operator. In [7] this decomposition was used in the case $\beta=0$ (in discrete time, see [6]) to extend the truth of the conjecture for normal semigroups and the right-shift semigroup to general contraction semigroups. Thus, it is disappointing that neither the right-shift semigroup on $L^{2}\left(\mathbb{R}_{+}\right)$nor the unilateral shift on $H^{2}(\mathbb{D})$ satisfy the weighted Weiss conjecture in the case $\beta \in(0,1)$.

The main results of this paper are to obtain positive results characterising $\beta$-admissibility for shift operators and semigroups. Results are proven in discrete time for the unilateral shift and in continuous time for the right-shift semigroup. For technical simplicity we first describe results in the discrete time setting. Two approaches are taken. The first is to consider the unilateral shift $(S f)(z)=z f(z)$ acting on a different space to $H^{2}(\mathbb{D})$. In Section $2, \beta$-admissibility of the shift $S: X \rightarrow X$ is considered in the case that $X$ is a weighted Bergman space $\mathcal{A}_{\alpha}^{2}(\mathbb{D}), \alpha>-1$, which contains analytic functions $f: \mathbb{D} \rightarrow \mathbb{C}$ for which

$$
\|f\|_{\mathcal{A}_{\alpha}^{2}(\mathbb{D})}^{2}=\int_{\mathbb{D}}|f(z)|^{2} d A_{\alpha}(z)<\infty
$$

where $d A_{\alpha}(z)=(1+\alpha)\left(1-|z|^{2}\right)^{\alpha} d A(z)$ and $d A(z):=\frac{1}{\pi} d x d y$ is area measure on the unit disc $\mathbb{D}=\{z \in \mathbb{C}:|z|=1\}$, for $z=x+i y$. Since the norm $\|f\|_{\mathcal{A}_{\alpha}^{2}(\mathbb{D})}$ is equivalent to

$$
\left(\sum_{n=0}^{\infty}\left|f_{n}\right|^{2}(1+n)^{-(1+\alpha)}\right)^{\frac{1}{2}}
$$

where $f_{n}$ are the Taylor coefficients of $f$, naively, the Hardy space $H^{2}(\mathbb{D})$ may be thought of as the 'corner' of the family of weighted Bergman spaces as $\alpha \rightarrow-1^{+}$. However, the behaviour of the weighted Weiss conjecture changes at this corner: it is shown in Theorem 2.9 that for $\beta>0$ the resolvent bound characterisation $(4) \Rightarrow(3)$ of $\beta$-admissibility holds for the shift $S: \mathcal{A}_{\alpha}^{2}(\mathbb{D}) \rightarrow$ $\mathcal{A}_{\alpha}^{2}(\mathbb{D})$, for any $\alpha>-1$. The second approach, taken in Section 4 , is to derive a modified resolvent growth bound characterisation of $\beta$-admissibility for the shift $S: H^{2}(\mathbb{D}) \rightarrow H^{2}(\mathbb{D})$. In this case, it is shown in Corollary 4.5 that $\beta$-admissibility is characterised by

$$
\sup _{\omega \in \mathbb{D}}\left(1-|\omega|^{2}\right)^{\frac{1}{2}}\left\|C(I-\bar{\omega} S)^{-1}\right\|_{\mathcal{A}_{\beta-1}^{2}(\mathbb{D})^{*}}<\infty .
$$

The difference between this condition and (4), which does not characterise $\beta$-admissibility of $S$ : $H^{2}(\mathbb{D}) \rightarrow H^{2}(\mathbb{D})$, is that the weight $\beta$ appears in the space in which the norm of the operator $C(I-\bar{\omega} S)^{-1}$ is tested, rather than as a power of the resolvent and the required growth rate.

That (6) is in some sense the 'correct' resolvent growth condition with which to test weighted admissibility of $S: H^{2}(\mathbb{D}) \rightarrow H^{2}(\mathbb{D})$ is intrinsically related to the notion of a Reproducing Kernel Thesis (RKT). A Reproducing Kernel Hilbert Space $H$ is a space of analytic functions on a set $\Omega$ (in this paper, either $\Omega=\mathbb{D}$ or $\Omega=\mathbb{C}_{+}=\{\lambda: \operatorname{Re} \lambda>0\}$ ) containing functions $\left(k_{\omega}\right)_{\omega \in \Omega} \subset H$, known as the reproducing kernels, which satisfy $f(\omega)=\left\langle f, k_{\omega}\right\rangle_{H}$ for any $f \in H, \omega \in \Omega$. A class $\mathcal{T}$ which contains linear operators acting from $H$ to a second Hilbert space $K$ is said to satisfy a Reproducing Kernel Thesis if boundedness of each operator $T \in \mathcal{T}$ is characterized by

$$
\sup _{\omega \in \Omega} \frac{\left\|T k_{\omega}\right\|_{K}}{\left\|k_{\omega}\right\|_{H}}<\infty .
$$


That is, for each operator $T \in \mathcal{T}$, boundedness of $T: H \rightarrow K$ can be tested just by considering the behaviour of $T$ on the reproducing kernels.

The question of which classes of operator satisfy a RKT has received much attention and it is known that many important classes of operator do satisfy a RKT (see, e.g. [11, p. 131] for a brief overview). Of particular relevance to the study of the Weiss conjecture for shifts is the fact that the class of little Hankel operators $h_{\bar{c}}(f):=\bar{P}(\bar{c} f)$ with symbol $c \in H^{2}(\mathbb{D})$, mapping from $H^{2}(\mathbb{D})$ to $\overline{H^{2}(\mathbb{D})}=\bar{P} H^{2}(\mathbb{D})$, satisfy a RKT. Here, $\bar{P}$ denotes the projection onto anti-analytic functions. In the case $\beta=0$, it was shown in [6] that if $T=S$ is the unilateral shift on $H^{2}(\mathbb{D})$ and $C f=\langle f, c\rangle_{H^{2}}$ for $c \in H^{2}(\mathbb{D})$, then $(3)$ holds if and only if $h_{\bar{c}}$ is bounded on $H^{2}(\mathbb{D})$. On the other hand, since the reproducing kernels for $H^{2}(\mathbb{D})$ are $k_{w}(z)=(1-\bar{\omega} z)^{-1}$ and $\left\|k_{\omega}\right\|_{H^{2}(\mathbb{D})}=\left(1-|\omega|^{2}\right)^{-\frac{1}{2}}$, it is not difficult to show that $h_{\bar{c}}: H^{2}(\mathbb{D}) \rightarrow \overline{H^{2}(\mathbb{D})}$ satisfies $(7)$ if and only if the resolvent condition (4) holds for $\beta=0$. Hence, the truth of the discrete Weiss conjecture for the shift $S$ in the unweighted case $\beta=0$ is equivalent to the fact that the Hankel operators $h_{\bar{c}}$, for $c \in H^{2}(\mathbb{D})$, satisfy a RKT.

In the weighted case $\beta>0$, it is shown in Propositions 4.2 and 4.3 that $\beta$-admissibility of the shift $S: H^{2}(\mathbb{D}) \rightarrow H^{2}(\mathbb{D})$ is equivalent to boundedness of one/both of the generalized Hankel operators $h_{\bar{c}}^{\beta / 2,0}$ or $h_{\bar{c}}^{0, \beta / 2}$ (definitions of these operators are given in Section 4.1). It turns out that whether $h_{\bar{c}}^{\beta / 2,0}$ satisfies (7) is equivalent to the modified resolvent condition (6); while whether $h_{\bar{c}}^{0, \beta / 2}$ satisfies (7) is equivalent to the original resolvent condition (4). Consequently, the characterization $(3) \Leftrightarrow(6)$ of $\beta$-admissibility follows from the fact that the generalized Hankel operators $h_{\bar{c}}^{\beta / 2,0}$, for $c \in H^{2}(\mathbb{D})$, satisfy a RKT (proven in Theorem 4.4); while the failure of the original conjecture $(4) \nRightarrow(3)$ can now be explained by the fact that the operators $h_{\bar{c}}^{0, \beta / 2}$, for $c \in H^{2}(\mathbb{D})$, do not. The technical reason for this result is that the inclusion $D^{-\beta / 2} B M O A \subset \Lambda_{\beta / 2}^{+}$ between two certain classes of operator symbols is strict.

Analogous results to the ones described above are proven for the continuous time case. In Section 3, Theorem 3.1, it is shown that for $\beta>0$ the weighted Weiss conjecture $(2) \Rightarrow(1)$ holds for the right-shift semigroup acting on any of the weighted spaces $L_{\alpha}^{2}\left(\mathbb{R}_{+}\right), \alpha>0$, where

$$
L_{\alpha}^{2}\left(\mathbb{R}_{+}\right):=\left\{f: \mathbb{R}_{+} \rightarrow \mathbb{C}: f \text { measurable, } \int_{0}^{\infty} t^{-\alpha}|f(t)|^{2} d t<\infty\right\}
$$

The 'corner' case of the right-shift semigroup on $L^{2}\left(\mathbb{R}_{+}\right)$is discussed in Section 5 , where it is shown in Corollary 5.9 that $\beta$-admissibility, $\beta>0$, is characterised by the modified resolvent growth condition

$$
\sup _{\lambda \in \mathbb{C}_{+}}(\operatorname{Re} \lambda)^{\frac{1}{2}}\|C R(\lambda, A)\|_{L_{\beta / 2}^{2}\left(\mathbb{R}_{+}\right)^{*}}<\infty
$$

where $A$ is the generator of the right-shift semigroup. In the continuous time setting, the characterisation of weighted admissibility is related to whether certain generalised Hankel operators satisfy a RKT on the Hardy space $H^{2}\left(\mathbb{C}_{+}\right)$.

\section{Discrete time $\beta$-admissibility of the unilateral shift on weighted Bergman spaces}

In this section, discrete-time $\beta$-admissibility is studied for the unilateral shift $S: \mathcal{A}_{\alpha}^{2}(\mathbb{D}) \rightarrow \mathcal{A}_{\alpha}^{2}(\mathbb{D})$ given by

$$
(S f)(z)=z f(z), \quad f \in \mathcal{A}_{\alpha}^{2}(\mathbb{D}) .
$$

In the following, the inner product for $\mathcal{A}_{\alpha}^{2}(\mathbb{D})$ is written $\langle,\rangle_{\alpha}$. 
2.1 Equivalent conditions for discrete $\beta$-admissibility and the resolvent bound (4).

First, $\beta$-admissibility of an observation functional $C \in \mathcal{A}_{\alpha}^{2}(\mathbb{D})^{*}$ is characterized in terms of its associated Taylor coefficients.

Proposition 2.1. Let $\alpha>-1, \beta>0$. Suppose that $C \in \mathcal{A}_{\alpha}^{2}(\mathbb{D})^{*}$ is given by $C f:=\langle f, c\rangle_{\alpha}$, where $c=\sum_{n=0}^{\infty} c_{n} z^{n} \in \mathcal{A}_{\alpha}^{2}(\mathbb{D})$. Then $C$ is discrete $\beta$-admissible for $S$ if and only if

$$
\sum_{n=0}^{\infty}\left|\sum_{m=0}^{\infty} \frac{(1+n)^{\frac{\beta}{2}}}{(1+n+m)^{1+\alpha}} f_{m} \bar{c}_{n+m}\right|^{2} \leq k\|f\|_{\mathcal{A}_{\alpha}^{2}(\mathbb{D})}^{2}, \quad f \in \mathcal{A}_{\alpha}^{2}(\mathbb{D})
$$

Proof. For $f \in \mathcal{A}_{\alpha}^{2}(\mathbb{D})$,

$$
\begin{aligned}
\sum_{n=0}^{\infty}(1+n)^{\beta}\left|C S^{n} f\right|^{2} & =\sum_{n=0}^{\infty}(1+n)^{\beta}\left|\left\langle\sum_{m=0}^{\infty} f_{m} z^{m+n}, \sum_{m=0}^{\infty} c_{m} z^{m}\right\rangle_{\alpha}\right|^{2} \\
& =\sum_{n=0}^{\infty}(1+n)^{\beta}\left|\left\langle\sum_{m=n}^{\infty} f_{m-n} z^{m}, \sum_{m=0}^{\infty} c_{m} z^{m}\right\rangle_{\alpha}\right|^{2} \\
& =\sum_{n=0}^{\infty}(1+n)^{\beta}\left|\sum_{m=n}^{\infty} f_{m-n} \bar{c}_{m}(1+m)^{-(1+\alpha)}\right|^{2} \\
& =\sum_{n=0}^{\infty}\left|\sum_{m=0}^{\infty} \frac{(1+n)^{\frac{\beta}{2}}}{(1+n+m)^{1+\alpha}} f_{m} \bar{c}_{n+m}\right|^{2} \cdot
\end{aligned}
$$

In order to form a comparable expression for the resolvent condition (4), it is necessary to define the operator $g(S)$ for suitable functions $g$. To this end, let

$$
\mathcal{O}(\overline{\mathbb{D}}):=\left\{g \in H(\mathbb{D}): \exists \nu>1 \text { such that } \sum_{n=0}^{\infty}\left|g_{n}\right| \nu^{n}<\infty\right\}
$$

where $g$ has Taylor series $g(z)=\sum_{n=0}^{\infty} g_{n} z^{n}$. Then by [6, Lemma 2.1], $g(S) \in \mathcal{L}(X)$ for any $g \in \mathcal{O}(\bar{D})$. Note that $g(z)=(1-\bar{\omega} z)^{-(1+\beta)} \in \mathcal{O}(\overline{\mathbb{D}})$ for each $\omega \in \mathbb{D}$.

Proposition 2.2. Let $\alpha>-1$. Suppose that $C \in \mathcal{A}_{\alpha}^{2}(\mathbb{D})^{*}$ is given by $C f:=\langle f, c\rangle_{\alpha}$, where $c=$ $\sum_{n=0}^{\infty} c_{n} z^{n} \in \mathcal{A}_{\alpha}^{2}(\mathbb{D})$. Then for any $g=\sum_{n=0}^{\infty} g_{n} z^{n} \in \mathcal{O}(\overline{\mathbb{D}})$,

$$
\|C g(S)\|_{\mathcal{A}_{\alpha}^{2}(\mathbb{D})^{*}}^{2}=\sum_{n=0}^{\infty}\left|\sum_{m=0}^{\infty} \frac{(1+n)^{\frac{1+\alpha}{2}}}{(1+n+m)^{1+\alpha}} g_{m} \bar{c}_{n+m}\right|^{2} .
$$


Proof. For $f \in \mathcal{A}_{\alpha}^{2}(\mathbb{D})$,

$$
\begin{aligned}
C g(S) f & =\langle g(S) f, c\rangle_{\alpha} \\
& =\left\langle\sum_{m=0}^{\infty}\left(\sum_{n=0}^{m} f_{n} g_{m-n}\right) z^{m}, \sum_{m=0}^{\infty} c_{m} z^{m}\right\rangle_{\alpha} \\
& =\sum_{m=0}^{\infty}(1+m)^{-(1+\alpha)}\left(\sum_{n=0}^{m} f_{n} g_{m-n}\right) \bar{c}_{m} \\
& =\sum_{n=0}^{\infty} \sum_{m=n}^{\infty}(1+m)^{-(1+\alpha)} f_{n} g_{m-n} \bar{c}_{m} \\
& =\sum_{n=0}^{\infty}(1+n)^{-(1+\alpha)}\left(\sum_{m=0}^{\infty} \frac{(1+n)^{1+\alpha}}{(1+m+n)^{1+\alpha}} g_{m} \bar{c}_{n+m}\right) f_{n} \\
& =\langle f, h\rangle_{\alpha},
\end{aligned}
$$

where

$$
h(z)=\sum_{n=0}^{\infty}\left(\sum_{m=0}^{\infty} \frac{(1+n)^{1+\alpha}}{(1+m+n)^{1+\alpha}} \bar{g}_{m} c_{n+m}\right) z^{n} .
$$

Therefore,

$$
\begin{aligned}
\|C g(S)\|_{\mathcal{A}_{\alpha}^{2}(\mathbb{D})^{*}}^{2}=\|h\|_{\mathcal{A}_{\alpha}^{2}(\mathbb{D})}^{2} & =\sum_{n=0}^{\infty}(1+n)^{-(1+\alpha)}\left|\sum_{m=0}^{\infty} \frac{(1+n)^{1+\alpha}}{(1+m+n)^{1+\alpha}} \bar{g}_{m} c_{n+m}\right|^{2} \\
& =\sum_{n=0}^{\infty}\left|\sum_{m=0}^{\infty} \frac{(1+n)^{\frac{1+\alpha}{2}}}{(1+m+n)^{1+\alpha}} g_{m} \bar{c}_{n+m}\right|^{2} \cdot \square
\end{aligned}
$$

2.2 Discrete $\beta$-admissibility and the little Hankel operator.

The link between Hankel operators and admissibility has previously been frequently exploited [6, $7,12,22]$ in order to study admissibility. In this section, it is shown that boundedness of little Hankel operators between weighted Bergman spaces characterise weighted admissibility of $S$ on weighted Bergman spaces.

The little Hankel operator $h_{f}: \mathcal{A}_{\alpha}^{2}(\mathbb{D}) \rightarrow \overline{\mathcal{A}_{\alpha}^{2}(\mathbb{D})}$ is defined by

$$
h_{f} g=\overline{P_{\alpha}}(f g)
$$

where $\overline{P_{\alpha}}$ is the orthogonal projection from $L^{2}\left(\mathbb{D},\left(1-|z|^{2}\right)^{\alpha} d A\right)$ to the space of anti-analytic functions

$$
\overline{\mathcal{A}_{\alpha}^{2}(\mathbb{D})}:=\left\{\bar{f}: f \in \mathcal{A}_{\alpha}^{2}(\mathbb{D})\right\} .
$$

Suppose that $f \in \mathcal{A}_{\alpha}^{2}(\mathbb{D})$. Then using the fact that $\left\{\bar{z}^{n}, n=0,1,2, \ldots\right\}$ is a basis for $\overline{\mathcal{A}_{\alpha}^{2}(\mathbb{D})}$,

$$
\begin{aligned}
\left\langle h_{\bar{f}} z^{m}, \bar{z}^{n}\right\rangle_{\alpha} & =\left\langle\overline{P_{\alpha}}\left(\bar{f}(z) z^{m}\right), \bar{z}^{n}\right\rangle_{\alpha} \\
& =\left\langle\bar{f}(z) z^{m}, \bar{z}^{n}\right\rangle_{\alpha} \\
& =\left\langle\bar{f}(z), \bar{z}^{m+n}\right\rangle_{\alpha} \\
& =\bar{f}_{n+m}\left\langle\bar{z}^{m+n}, \bar{z}^{m+n}\right\rangle_{\alpha} \\
& =\frac{\bar{f}_{n+m}}{(1+n+m)^{1+\alpha}} .
\end{aligned}
$$


Therefore, if $g=\sum_{m=0}^{\infty} g_{m} z^{m}$,

$$
\left\langle h_{\bar{f}} g, \bar{z}^{n}\right\rangle_{\alpha}=\sum_{m=0}^{\infty} \frac{g_{m} \bar{f}_{n+m}}{(1+n+m)^{1+\alpha}} .
$$

and since $\left\langle\bar{z}^{n}, \bar{z}^{n}\right\rangle_{\alpha}=(1+n)^{-(1+\alpha)}$, it follows that

$$
\left(h_{\bar{f}} g\right)_{n}=(1+n)^{1+\alpha} \sum_{m=0}^{\infty} \frac{g_{m} \bar{f}_{n+m}}{(1+n+m)^{1+\alpha}},
$$

where $\left(h_{\bar{f}} g\right)_{n}$ is the $n^{t h}$ Fourier coefficient with respect to the basis $\left\{\bar{z}^{n}, n=0,1,2, \ldots\right\}$. Hence,

$$
\left\|h_{\bar{f}} g\right\|_{\frac{2}{\mathcal{A}_{\alpha}^{2}(\mathbb{D})}}=\sum_{n=0}^{\infty}\left|\sum_{m=0}^{\infty} \frac{(1+n)^{\frac{1+\alpha}{2}}}{(1+n+m)^{1+\alpha}} g_{m} \bar{f}_{n+m}\right|^{2} .
$$

Therefore, there is a link between weighted admissibility on weighted Bergman spaces, and boundedness of the little Hankel operator on weighted Bergman spaces. In the following,

$$
k_{\omega}^{\alpha}(z):=\frac{\left(1-|\omega|^{2}\right)^{1+\frac{\alpha}{2}}}{(1-\bar{\omega} z)^{2+\alpha}}, \quad \omega \in \mathbb{D}, z \in \mathbb{D}
$$

are the normalized reproducing kernels for $\mathcal{A}_{\alpha}^{2}(\mathbb{D})$.

Proposition 2.3. Let $\alpha>-1, \beta>0$. Suppose that $C \in \mathcal{A}_{\alpha}^{2}(\mathbb{D})^{*}$ is given by $C f:=\langle f, c\rangle_{\alpha}$, where $c=\sum_{n=0}^{\infty} c_{n} z^{n} \in \mathcal{A}_{\alpha}^{2}(\mathbb{D})$. Then

(i) $C$ is discrete $\beta$-admissible for $S$ if and only if $h_{\bar{c}}: \mathcal{A}_{\beta-1}^{2}(\mathbb{D}) \rightarrow \overline{\mathcal{A}_{\alpha}^{2}(\mathbb{D})}$ is bounded.

(ii) For any $\omega \in \mathbb{D}$,

$$
\left(1-|\omega|^{2}\right)^{\frac{1+\beta}{2}}\left\|C(I-\bar{\omega} S)^{-(1+\beta)}\right\|_{\mathcal{A}_{\alpha}^{2}(\mathbb{D})^{*}}=\left\|h_{\bar{c}} k_{\omega}^{\beta-1}\right\|_{\overline{\mathcal{A}_{\alpha}^{2}(\mathbb{D})}} .
$$

Proof. (i) By Proposition 2.1, and the equivalent expression (5) for norm $\|\cdot\|_{\mathcal{A}_{\alpha}^{2}(\mathbb{D})}$, $C$ is discrete $\beta$-admissible for $S$ if and only if the matrix $A=\left(a_{n m}\right)$ with coefficients

$$
a_{n m}=\frac{(1+m)^{\frac{1+\alpha}{2}}(1+n)^{\frac{1+(\beta-1)}{2}}}{(1+n+m)^{1+\alpha}} \bar{c}_{n+m},
$$

is bounded from $\ell^{2}$ to $\ell^{2}$. On the other hand, $h_{\bar{c}}: \mathcal{A}_{\beta-1}^{2}(\mathbb{D}) \rightarrow \overline{\mathcal{A}_{\alpha}^{2}(\mathbb{D})}$ is bounded if and only if

$$
\left\|h_{\bar{c}} g\right\|_{\overline{\mathcal{A}_{\alpha}^{2}(\mathbb{D})}} \leq k\|g\|_{\mathcal{A}_{\beta-1}^{2}(\mathbb{D})}, \quad g \in \mathcal{A}_{\beta-1}^{2}(\mathbb{D}),
$$

which by (9) and (5) occurs if and only if $A: \ell^{2} \rightarrow \ell^{2}$ is bounded.

(ii) Follows from Proposition 2.2, (9) and the fact that $k_{\omega}^{\beta-1} \in \mathcal{O}(\overline{\mathbb{D}})$ for each $\omega \in \mathbb{D}$.

Proposition 2.3 implies that the question of whether the discrete weighted Weiss conjecture $(3) \Leftrightarrow(4)$ holds for $S: \mathcal{A}_{\alpha}^{2}(\mathbb{D}) \rightarrow \mathcal{A}_{\alpha}^{2}(\mathbb{D})$ in the case $\beta>0$ is equivalent to the following question: does the Hankel operator $h_{\bar{c}}: \mathcal{A}_{\beta-1}^{2}(\mathbb{D}) \rightarrow \overline{\mathcal{A}_{\alpha}^{2}(\mathbb{D})}$ satisfy a RKT? Specifically, does (7) with $T=h_{\bar{c}}, H=\mathcal{A}_{\beta-1}^{2}(\mathbb{D})$ and $K=\overline{\mathcal{A}_{\alpha}^{2}(\mathbb{D})}$ imply boundedness of $h_{\bar{c}}$ ?

It is shown in [24, Theorem 8.39] that the question has a positive answer in the case $h_{\bar{c}}$ : $\mathcal{A}_{\alpha}^{2}(\mathbb{D}) \rightarrow \overline{\mathcal{A}_{\alpha}^{2}(\mathbb{D})}$. In fact the method of proof can be adapted to show that $h_{\bar{c}}: \mathcal{A}_{\gamma}^{2}(\mathbb{D}) \rightarrow \overline{\mathcal{A}_{\alpha}^{2}(\mathbb{D})}$ 
also satisfies RKT for any $\alpha, \gamma>-1$. For $\alpha, \gamma>-1$, and $f \in L^{2}\left(\mathbb{D}, d A_{\alpha}\right)$ define the integral operator

$$
(V f)(z):=\left(\frac{3+\alpha+\gamma}{1+\alpha}\right)\left(1-|z|^{2}\right)^{2+\gamma} \int_{\mathbb{D}} \frac{f(w)}{(1-z \bar{\omega})^{4+\alpha+\gamma}} d A_{\alpha}(\omega), \quad z \in \mathbb{D}
$$

and the projection $P_{\alpha}: L^{2}\left(\mathbb{D}, d A_{\alpha}\right) \rightarrow \mathcal{A}_{\alpha}^{2}(\mathbb{D})$ by

$$
\left(P_{\alpha} f\right)(z)=\int_{\mathbb{D}} \frac{f(w)}{(1-z \bar{\omega})^{2+\alpha}} d A_{\alpha}(w) .
$$

The following properties of $V$ can now be deduced.

Lemma 2.4. Suppose that $\alpha, \gamma>-1$. Then

(i) The operator $V$ is bounded on $L^{2}\left(\mathbb{D}, d A_{\alpha}\right)$;

(ii) $P_{\alpha} V=P_{\alpha}$.

Proof. (i) By [Zhu, Theorem 3.11], $V=T_{2+\gamma, \alpha, 4+\alpha+\gamma}$, which is bounded on $L^{2}\left(\mathbb{D}, d A_{\alpha}\right)$.

(ii) For $f \in L^{2}\left(\mathbb{D}, d A_{\alpha}\right)$,

$$
\begin{aligned}
& \left(P_{\alpha} V f\right)(z)=\int_{\mathbb{D}} \frac{(V f)(w)}{(1-z \bar{\omega})^{2+\alpha}} d A_{\alpha}(\omega) \\
& =\left(\frac{3+\alpha+\gamma}{1+\alpha}\right) \int_{\mathbb{D}} \frac{\left(1-|\omega|^{2}\right)^{2+\gamma}}{(1-z \bar{\omega})^{2+\alpha}} \int_{\mathbb{D}} \frac{f(u)}{(1-w \bar{u})^{4+\alpha+\gamma}} d A_{\alpha}(u) d A_{\alpha}(w) \\
& =\left(\frac{3+\alpha+\gamma}{1+\alpha}\right) \int_{\mathbb{D}}\left(\int_{\mathbb{D}} \frac{\left(1-|\omega|^{2}\right)^{2+\gamma}}{(1-z \bar{\omega})^{2+\alpha}(1-\omega \bar{u})^{4+\alpha+\gamma}} d A_{\alpha}(\omega)\right) f(u) d A_{\alpha}(u) \\
& =\int_{\mathbb{D}}\left(\overline{\int_{\mathbb{D}} \frac{d A_{2+\alpha+\gamma}(\omega)}{(1-\bar{z} \omega)^{2+\alpha}(1-\bar{\omega} u)^{4+\alpha+\gamma}}}\right) f(u) d A_{\alpha}(u) \\
& =\int_{\mathbb{D}} \frac{1}{(1-\bar{u} z)^{2+\alpha}} f(u) d A_{\alpha}(u) \\
& =\int_{\mathbb{D}} \frac{f(u)}{(1-z \bar{u})^{2+\alpha}} d A_{\alpha}(u) \\
& =\left(P_{\alpha} f\right)(z) \text {. }
\end{aligned}
$$

Lemma 2.5. Let $\alpha, \gamma>-1$. If $\left(1-|z|^{2}\right)^{\frac{\alpha-\gamma}{2}} f(z) \in L^{\infty}(\mathbb{D})$, then

$$
h_{f} \in \mathcal{L}\left(\mathcal{A}_{\gamma}^{2}(\mathbb{D}), \overline{\mathcal{A}_{\alpha}^{2}(\mathbb{D})}\right) .
$$

Proof. For $g \in \mathcal{A}_{\gamma}^{2}(\mathbb{D})$,

$$
\begin{aligned}
\left\|h_{f} g\right\|_{\overline{\mathcal{A}_{\alpha}^{2}(\mathbb{D})}} & =\left\|\overline{P_{\alpha}}(f g)\right\|_{\overline{\mathcal{A}_{\alpha}^{2}(\mathbb{D})}} \\
& \leq\|f g\|_{L^{2}\left(\mathbb{D}, d A_{\alpha}\right)} \\
& =\left((1+\alpha) \int_{\mathbb{D}}|f(z)|^{2}|g(z)|^{2}\left(1-|z|^{2}\right)^{\alpha} d A(z)\right)^{\frac{1}{2}} \\
\text { (by assumption) } & \leq k\left(\int_{\mathbb{D}}|g(z)|^{2}\left(1-|z|^{2}\right)^{\gamma} d A(z)\right)^{\frac{1}{2}}=k\|g\|_{\mathcal{A}_{\gamma}^{2}(\mathbb{D}) .}
\end{aligned}
$$


The action of the little Hankel operator on reproducing kernels is now related to the integral operator $V$.

Lemma 2.6. Let $\alpha, \gamma>-1$. Then for $f \in L^{2}\left(\mathbb{D}, d A_{\alpha}\right)$,

$$
\left\langle\bar{k}_{z}^{\alpha}, h_{\bar{f}} k_{z}^{\gamma}\right\rangle_{\alpha}=\frac{(1+\alpha)}{(3+\alpha+\gamma)}\left(1-|z|^{2}\right)^{\frac{\alpha-\gamma}{2}}(V f)(z), \quad z \in \mathbb{D}
$$

Proof. Using the fact that $\overline{h_{\bar{f}} k_{z}^{\gamma}}$ is analytic,

$$
\begin{aligned}
\left\langle\bar{k}_{z}^{\alpha}, h_{\bar{f}} k_{z}^{\gamma}\right\rangle_{\alpha} & =\left\langle\overline{h_{\bar{f}} k_{z}^{\gamma}}, k_{z}^{\alpha}\right\rangle_{\alpha} \\
& =\left(1-|z|^{2}\right)^{1+\frac{\alpha}{2}} \overline{h_{\bar{f}} k_{z}^{\gamma}}(z) \\
& =\left(1-|z|^{2}\right)^{1+\frac{\alpha}{2}} \int_{\mathbb{D}} \frac{\overline{f(\omega)} k_{z}^{\gamma}(\omega)}{(1-\bar{z} \omega)^{2+\alpha}} d A_{\alpha}(\omega) \\
& =\left(1-|z|^{2}\right)^{2+\frac{\alpha+\gamma}{2}} \int_{\mathbb{D}} \frac{f(\omega)}{(1-z \bar{\omega})^{4+\alpha+\gamma}} d A_{\alpha}(\omega) \\
& =\frac{(1+\alpha)}{(3+\alpha+\gamma)}\left(1-|z|^{2}\right)^{\frac{\alpha-\gamma}{2}}(V f)(z) .
\end{aligned}
$$

As a consequence, the little Hankel operators $h_{\bar{f}}: \mathcal{A}_{\gamma}^{2}(\mathbb{D}) \rightarrow \overline{\mathcal{A}_{\alpha}^{2}(\mathbb{D})}$ satisfy the reproducing kernel thesis.

Theorem 2.7. Let $\alpha, \gamma>-1$. Then $\sup \left\{\left\|h_{\bar{f}} k_{z}^{\gamma}\right\|_{\overline{\mathcal{A}_{\alpha}^{2}(\mathbb{D})}}: z \in \mathbb{D}\right\}<\infty$ if and only if the little Hankel operator $h_{\bar{f}}: \mathcal{A}_{\gamma}^{2}(\mathbb{D}) \rightarrow \overline{\mathcal{A}_{\alpha}^{2}(\mathbb{D})}$ is bounded.

Proof. If $f \in L^{2}\left(\mathbb{D}, d A_{\alpha}\right)$ then by Lemma 2.4 ,

$$
h_{\bar{f}}=h_{\overline{P_{\alpha} f}}=h_{\overline{P_{\alpha} V f}}=h_{\overline{V f}} .
$$

By assumption and Lemma 2.6,

$$
\frac{(1+\alpha)}{(3+\alpha+\gamma)}\left(1-|z|^{2}\right)^{\frac{\alpha-\gamma}{2}}|(V f)(z)|=\left|\left\langle\bar{k}_{z}^{\alpha}, h_{\bar{f}} k_{z}^{\gamma}\right\rangle_{\alpha}\right| \leq\left\|h_{\bar{f}} k_{z}^{\gamma}\right\|_{L^{2}\left(\mathbb{D}, d A_{\alpha}\right)}<k, \quad z \in \mathbb{D} .
$$

By (10) and Lemma $2.5, h_{\bar{f}}$ is bounded.

Remark 2.8. It should be noted that boundedness of the little Hankel operator $h_{\bar{f}}: \mathcal{A}_{\gamma}^{2}(\mathbb{D}) \rightarrow \overline{\mathcal{A}_{\alpha}^{2}(\mathbb{D})}$ has been characterised, in terms of symbols, in [8]. Theorem 2.7 therefore provides an additional characterisation of boundedness.

Using Proposition 2.3 and Theorem 2.7, we can prove that the weighted Weiss conjecture is true for the shift on weighted Bergman spaces.

Theorem 2.9. Let $\alpha>-1, \beta>0$. Suppose that $C \in \mathcal{A}_{\alpha}^{2}(\mathbb{D})^{*}$ is given by $C f:=\langle f, c\rangle_{\alpha}$, where $c=\sum_{n=0}^{\infty} c_{n} z^{n} \in \mathcal{A}_{\alpha}^{2}(\mathbb{D})$. Then $C$ is discrete $\beta$-admissible for $S$ if and only if

$$
\sup _{\omega \in \mathbb{D}}\left(1-|\omega|^{2}\right)^{\frac{1+\beta}{2}}\left\|C(I-\bar{\omega} S)^{-(1+\beta)}\right\|_{\mathcal{A}_{\alpha}^{2}(\mathbb{D})^{*}}<\infty .
$$




\section{$3 \beta$-admissibility of the right-shift semigroup on $L_{\alpha}^{2}\left(\mathbb{R}_{+}\right)$}

In this section Theorem 2.9 is translated to continuous time for the right-shift $C_{0}$-semigroup on $L_{\alpha}^{2}\left(\mathbb{R}_{+}\right)$given by

$$
(S(t) f)(\tau):=\left\{\begin{array}{cc}
f(\tau-t), & \tau \geq t ; \\
0, & \tau<t
\end{array} \quad t \geq 0, f \in L_{\alpha}^{2}\left(\mathbb{R}_{+}\right)\right.
$$

Up to a constant, the Laplace transform $\mathcal{L}: L_{\alpha}^{2}\left(\mathbb{R}_{+}\right) \rightarrow \mathcal{A}_{\alpha-1}^{2}\left(\mathbb{C}_{+}\right)$is an isometric isomorphism [1]. Here, for each $\gamma>-1$,

$$
\mathcal{A}_{\gamma}^{2}\left(\mathbb{C}_{+}\right):=\left\{F: \mathbb{C}_{+} \rightarrow \mathbb{C}:\|F\|_{\mathcal{A}_{\gamma}^{2}\left(\mathbb{C}_{+}\right)}^{2}:=\int_{-\infty}^{\infty} \int_{0}^{\infty} x^{\gamma}|F(x+i y)|^{2} d x d y<\infty\right\}
$$

is the weighted Bergman space on the right-half-plane $\mathbb{C}_{+}$. Under the isomorphism provided by the Laplace transform, $(S(t))_{t \geq 0}$ is equivalent to the semigroup

$$
(T(t) f)(z)=e^{-z t} f(z), \quad f \in \mathcal{A}_{\alpha-1}^{2}\left(\mathbb{C}_{+}\right), z \in \mathbb{C}_{+}
$$

on the Bergman space $\mathcal{A}_{\alpha-1}^{2}\left(\mathbb{C}_{+}\right)$. Using this link, Theorem 2.9 can now be translated from discrete to continuous time.

Theorem 3.1. Let $\alpha, \beta>0$. Let $A$ be the generator of the right-shift semigroup $(S(t))_{t \geq 0}$ on $L_{\alpha}^{2}\left(\mathbb{R}_{+}\right)$. Then an observation operator $C \in \mathcal{L}(D(A), \mathbb{C})$ is $\beta$-admissible for $A$ if and only if

$$
\sup _{\lambda \in \mathbb{C}_{+}}(\operatorname{Re} \lambda)^{\frac{1+\beta}{2}}\left\|C R(\lambda, A)^{1+\beta}\right\|_{L_{\alpha}^{2}\left(\mathbb{R}_{+}\right)^{*}}<\infty
$$

Proof. Let

$$
\left(J_{\alpha} f\right)(z):=\frac{c_{\alpha}}{(1+z)^{1+\alpha}} f\left(\frac{1-z}{1+z}\right), \quad f \in \mathcal{A}_{\alpha}\left(\mathbb{C}_{+}\right), z \in \mathbb{D}
$$

be the isometric isomorphism $J_{\alpha}: \mathcal{A}_{\alpha}^{2}\left(\mathbb{C}_{+}\right) \rightarrow \mathcal{A}_{\alpha}^{2}(\mathbb{D})$. Then

$$
\Phi:=J_{\alpha-1} \circ \mathcal{L}: L_{\alpha}^{2}\left(\mathbb{R}_{+}\right) \rightarrow \mathcal{A}_{\alpha-1}^{2}(\mathbb{D})
$$

is also an isometric isomorphism, under which the shift semigroup $(S(t))_{t \geq 0}$ on $L_{\alpha}^{2}\left(\mathbb{R}_{+}\right)$is equivalent to the semigroup

$$
(Q(t) f)(z):=e^{-\left(\frac{1-z}{1+z}\right) t} f(z), \quad f \in \mathcal{A}_{\alpha-1}^{2}(\mathbb{D}), z \in \mathbb{D} .
$$

Notice that the unilateral shift $S$ is the co-generator of $(Q(t))_{t \geq 0}$ on $\mathcal{A}_{\alpha-1}^{2}(\mathbb{D})$.

Given $C \in \mathcal{L}(D(A), \mathbb{C})$, define an observation operator by $\tilde{C}:=C \Phi^{-1}$. If $\tilde{A}$ is the generator of $(Q(t))_{t \geq 0}$, then by assumption and the fact that $R(\lambda, \tilde{A})^{1+\beta}=\Phi R(\lambda, A)^{1+\beta} \Phi^{-1}$,

$$
\sup _{\lambda \in \mathbb{C}_{+}}(\operatorname{Re} \lambda)^{\frac{1+\beta}{2}}\left\|\tilde{C} R(\lambda, \tilde{A})^{1+\beta}\right\|_{\mathcal{A}_{\alpha-1}^{2}(\mathbb{D})^{*}}<\infty .
$$

By the above equation and an argument from [21], it follows that if $D:=\tilde{C}(I-\tilde{A})^{-(1+\beta)}$ then

$$
\sup _{\omega \in \mathbb{D}}\left(1-|\omega|^{2}\right)^{\frac{1+\beta}{2}}\left\|D(I-\bar{\omega} S)^{-(1+\beta)}\right\|_{\mathcal{A}_{\alpha-1}^{2}(\mathbb{D})^{*}}<\infty
$$

and Theorem 2.9 implies that $D$ is discrete $\beta$-admissible for $S$. It is shown in [21] that $D$ is discrete $\beta$-admissible for $S$ if and only if $\tilde{C}$ is $\beta$-admissible for $(Q(t))_{t \geq 0}$. Since $(Q(t))_{t \geq 0}$ and $(S(t))_{t \geq 0}$ are equivalent semigroups, it follows that $C$ is $\beta$-admissible for $(S(t))_{t \geq 0}$. 
4 Discrete $\beta$-admissibility of the unilateral shift on the Hardy space

In this section discrete $\beta$-admissibility, $\beta>0$, is characterised for the unilateral shift $S: H^{2}(\mathbb{D}) \rightarrow$ $H^{2}(\mathbb{D})$ given by $(S f)(z)=z f(z), z \in \mathbb{D}, f \in H^{2}(\mathbb{D})$. The Hardy space $H^{2}(\mathbb{D})$ is the set of complexvalued analytic functions $f(z)=\sum_{n=0}^{\infty} f_{n} z^{n}$ such that

$$
\|f\|_{H^{2}(\mathbb{D})}^{2}:=\sup _{0<r<1} \int_{\theta=0}^{2 \pi}\left|f\left(r e^{i \theta}\right)\right|^{2} d \theta=\sum_{n=0}^{\infty}\left|f_{n}\right|^{2}<\infty .
$$

The space $H^{2}(\mathbb{D})$ is a reproducing kernel space with the (non-normalized) reproducing kernel with respect to $w \in \mathbb{D}$ given by

$$
k_{w}(z)=\frac{1}{1-\bar{w} z}, \quad z \in \mathbb{D} .
$$

Note that $\left\|k_{w}\right\|_{H^{2}(\mathbb{D})}=\left(1-|w|^{2}\right)^{-\frac{1}{2}}$.

For a function $f \in \operatorname{Hol}(\mathbb{D})+\overline{\operatorname{Hol}(\mathbb{D})}$ we associate the sequence of Taylor coefficients $\left\{f_{n}\right\}_{n \in \mathbb{Z}} \subset$ $\mathbb{C}$ for which

$$
f(z)=\sum_{n=0}^{\infty} f_{n} z^{n}+\sum_{n=1}^{\infty} f_{-n} \bar{z}^{n}, \quad z \in \mathbb{D} .
$$

If $\left\{f_{n}\right\}_{n \in \mathbb{Z}}$ has finite support we say that $f$ is polynomial. The pairing between two functions $f, g \in \operatorname{Hol}(\mathbb{D})+\overline{\operatorname{Hol}(\mathbb{D})}$ is defined by

$$
\langle f, g\rangle=\sum_{n} f_{n} \overline{g_{n}}
$$

whenever the series converges. We are particularly interested in the cases when either both sequences are square summable, or one of the functions is polynomial. We will often work with $H^{2}(\mathbb{D})$ and $\overline{H^{2}(\mathbb{D})}$ as closed subspaces of $L^{2}(\mathbb{T})$, where the Taylor coefficients are interpreted as Fourier coefficients. Note that the pairing between $f, g \in L^{2}(\mathbb{T})$ coincides with the usual inner product.

We now introduce spaces of analytic functions which will be required to study discrete $\beta$ admissibility of the unilateral shift on $H^{2}(\mathbb{D})$.

For an integer $n \geq 1$, define the trigonometric polynomial $W_{n}$ by the Fourier coefficients

$$
\hat{W}_{n}(k)= \begin{cases}\frac{k-2^{n-1}}{2^{n-1}} & \text { if } k \in\left[2^{n-1}, 2^{n}\right), \\ \frac{2^{n+1}-k}{2^{n}} & \text { if } k \in\left[2^{n}, 2^{n+1}\right), \\ 0 & \text { otherwise. }\end{cases}
$$

For $n \leq-1$, let $W_{n}=\overline{W_{-n}}$, and finally $W_{0}(\theta)=e^{-i \theta}+1+e^{i \theta}$. For $s \in \mathbb{R}$, the Hölder-Zygmund space $\Lambda_{s}$ consists of distributions $f$ on $\mathbb{T}$ such that

$$
\|f\|_{\Lambda_{s}}=\sup _{n \in \mathbb{Z}} 2^{|n| s}\left\|W_{n} * f\right\|_{\infty}<\infty
$$

These spaces are introduced in [14, Appendix 2]. The parameter $s$ indicates in this way how quickly the Fourier coefficients of $f$ decay, and therefore the defining property of the HölderZygmund spaces is a smoothness condition. We will often consider the subspace $\Lambda_{s}^{+}$of holomorphic distributions in $\Lambda_{s}$. The space $\Lambda_{0}^{+}$is called the Bloch space.

Given a function $f \in L^{1}(\mathbb{T})$ we define the quantity

$$
\|f\|_{B M O}=\sup _{I \subset \mathbb{T}} \frac{1}{|I|} \int_{I}\left|f(t)-\frac{1}{|I|} \int_{I} f(s) d s\right| d t .
$$


We then define the space

$$
B M O A(\mathbb{D})=\left\{f \in H^{2}(\mathbb{D}):\|f\|_{B M O}<\infty\right\} .
$$

The space $B M O A(\mathbb{D})$ can be characterized using wavelets. Given a function $\psi: \mathbb{R} \rightarrow \mathbb{C}$ we let $\psi_{j}(x)=2^{j / 2} \psi\left(2^{j} x\right)$ for $j \in \mathbb{Z}$ and $\psi_{J}(x)=\psi_{j}\left(x-x_{J}\right)$ where $x_{J}$ is the left endpoint of the dyadic interval $J=\left[2 \pi k 2^{-j}, 2 \pi(k+1) 2^{-j}\right)$. We will need a function $\psi \in \mathcal{S}(\mathbb{R})$ such that

$$
\begin{aligned}
& \operatorname{supp} \hat{\psi} \subseteq\left\{\xi: \frac{1}{3} \leq|\xi| \leq \frac{4}{3}\right\}, \\
& \int_{-\infty}^{\infty} x^{k} \psi(x) d x=0, \quad k \in \mathbb{Z},
\end{aligned}
$$

and moreover is such that $\left\{\psi_{J}\right\}_{J}$, where $J$ ranges over all dyadic intervals, is an orthonormal basis for $L^{2}(\mathbb{R})$. Such a $\psi$ exists and is exemplified by the Littlewood-Paley wavelet constructed in $[10$, pp. 21-25, p. 75].

We identify $[0,2 \pi), \mathbb{R} / 2 \pi \mathbb{Z}$ and $\mathbb{T}$ with each other, using the mapping $x \mapsto e^{i x}$. For any dyadic interval $J \subseteq[0,2 \pi)$, we define the $2 \pi$-periodification of $\psi_{J}$ by

$$
\varphi_{J}(x)=\sum_{k \in \mathbb{Z}} \psi_{J}(x-2 \pi k), \quad x \in \mathbb{R} .
$$

The family $\left\{\varphi_{J}\right\}_{J \subseteq \mathbb{T}}$ dyadic together with the constant function 1 is an orthonormal wavelet on $\mathbb{T}$. Using this language we have the following proposition [10, page 162].

Proposition 4.1. A function $f \in H^{2}(\mathbb{D})$ is in BMOA if and only if there exists $M>0$ such that

$$
\sum_{J \subseteq I}\left|\left\langle f, \varphi_{J}\right\rangle\right|^{2} \leq M^{2}|I|
$$

for any dyadic interval $I \subseteq[0,2 \pi)$. Moreover inf $M \approx\|f\|_{B M O}$.

Let $\alpha \in \mathbb{R}$. In order to discuss weighted admissibility we introduce the following operator, defined for double sided sequences of numbers:

$$
D^{\alpha}:\left(a_{n}\right)_{n \in \mathbb{Z}} \mapsto\left((1+|n|)^{\alpha} a_{n}\right)_{n \in \mathbb{Z}}
$$

By letting $D^{\alpha}$ act on the sequence of Taylor coefficients of a function, $D^{\alpha}$ may be regarded as an operator acting on $\operatorname{Hol}(\mathbb{D})+\overline{\operatorname{Hol}(\mathbb{D})}$. Note that for $\alpha>0, D^{\alpha}: H^{2}(\mathbb{D}) \rightarrow \mathcal{A}_{2 \alpha-1}^{2}(\mathbb{D})$ isomorphically. For $\alpha, s \in \mathbb{R}$ we also have that $D^{\alpha} \Lambda_{s}=\Lambda_{s-\alpha}[14$, Equation (A2.15)]. It is well known the $B M O A \subset \Lambda_{0}^{+}$with strict inclusion and so it follows that $D^{-s} B M O A \subset \Lambda_{s}^{+}$with strict inclusion for all $s \in \mathbb{R}$.

4.1 Admissibility and the little Hankel operator on $H^{2}(\mathbb{D})$.

Given a function $f \in H^{2}(\mathbb{D})$, define the little Hankel operator $h_{\bar{f}}: H^{\infty}(\mathbb{D}) \rightarrow \overline{H^{2}(\mathbb{D})}$ by

$$
h_{\bar{f}} g=\bar{P}(\bar{f} g),
$$

where $\bar{P}$ is the orthogonal projection from $L^{2}(\mathbb{T})$ to $\overline{H^{2}(\mathbb{D})}$. We will investigate when this operator has a continuous extension $h_{\bar{f}}: H^{2}(\mathbb{D}) \rightarrow \overline{H^{2}(\mathbb{D})}$. 
In the same manner as before, we see that

$$
\begin{aligned}
\left\langle h_{\bar{f}} z^{m}, \bar{z}^{n}\right\rangle & =\left\langle\bar{P}\left(\bar{f}(z) z^{m}\right), \bar{z}^{n}\right\rangle \\
& =\left\langle\bar{f}(z) z^{m}, \bar{z}^{n}\right\rangle \\
& =\left\langle\bar{f}(z), \bar{z}^{m+n}\right\rangle \\
& =\bar{f}_{n+m}\left\langle\bar{z}^{m+n}, \bar{z}^{m+n}\right\rangle \\
& =\bar{f}_{n+m},
\end{aligned}
$$

so that the matrix for the operator $h_{\bar{f}}$, in the monomial bases $\left\{z^{n}: n=0,1,2, \ldots\right\}$ and $\left\{\bar{z}^{n}: n=\right.$ $0,1,2, \ldots\}$ in $H^{2}(\mathbb{D})$ and $\overline{H^{2}(\mathbb{D})}$ respectively, becomes

$$
\left\{\bar{f}_{n+m}\right\}_{n, m \geq 0}=\left(\begin{array}{cccc}
\bar{f}_{0} & \bar{f}_{1} & \bar{f}_{2} & \cdots \\
\bar{f}_{1} & \bar{f}_{2} & \bar{f}_{3} & \cdots \\
\bar{f}_{2} & \bar{f}_{3} & \bar{f}_{4} & \cdots \\
\vdots & \vdots & \vdots & \ddots
\end{array}\right)
$$

Define the generalized Hankel operator

$$
h_{\bar{f}}^{\alpha, \beta}: g \mapsto D^{\alpha} h_{\bar{f}} D^{\beta} g
$$

where $f \in H^{2}(\mathbb{D})$ and $\alpha, \beta \geq 0$. The operator is defined, at least for $g \in \mathcal{O}(\mathbb{D})$, in the sense that $h_{\bar{f}}^{\alpha, \beta} g \in \overline{A_{2 \alpha-1}^{2}(\mathbb{D})}$. The operator can be represented by the generalized Hankel matrix

$$
\left\{(1+n)^{\alpha}(1+m)^{\beta} \bar{f}_{n+m}\right\}_{n, m \geq 0}=\left(\begin{array}{cccc}
\bar{f}_{0} & 2^{\beta} \bar{f}_{1} & 3^{\beta} \bar{f}_{2} & \ldots \\
2^{\alpha} \bar{f}_{1} & 2^{\alpha} 2^{\beta} \bar{f}_{2} & 2^{\alpha} 3^{\beta} \bar{f}_{3} & \ldots \\
3^{\alpha} \bar{f}_{2} & 3^{\alpha} 2^{\beta} \bar{f}_{3} & 3^{\alpha} 3^{\beta} \bar{f}_{4} & \ldots \\
\vdots & \vdots & \vdots & \ddots
\end{array}\right) .
$$

The following proposition links the generalized Hankel operator to weighted admissibility.

Proposition 4.2. Let $C \in H^{2}(\mathbb{D})^{*}$, and let $c \in H^{2}(\mathbb{D})$ be given by $C f=\langle f, c\rangle$. If $\beta \geq 0$, then $C$ is discrete $2 \beta$-admissible for $S$ if and only $h_{\bar{c}}^{\beta, 0}: H^{2}(\mathbb{D}) \rightarrow \overline{H^{2}(\mathbb{D})}$ is bounded.

Proof. Observe that

$$
(1+n)^{\beta} C S^{n} f=(1+n)^{\beta} \sum_{m=0}^{\infty} f_{m} \bar{c}_{n+m}=(1+n)^{\beta}\left(h_{\bar{c}} f\right)_{n}=\left(h_{\bar{c}}^{\beta, 0} f\right)_{n} .
$$

Parseval's identity now completes the proof:

$$
\sum_{n=0}^{\infty}(1+n)^{2 \beta}\left|C S^{n} f\right|^{2}=\sum_{n=0}^{\infty}\left|\left(h_{\bar{c}}^{\beta, 0} f\right)_{n}\right|^{2}=\left\|h_{\bar{c}}^{\beta, 0} f\right\|_{H^{2}(\mathbb{D})}^{2} .
$$

The boundedness of the operators $h_{\bar{f}}^{\alpha, \beta}$ has been characterized in [9] and [13]. The results have been collected in [14, Chapter 6.8].

Proposition 4.3. Let $f \in H^{2}(\mathbb{D})$. With the notation above we have that:

(i) Let $\beta \geq 0$. Then each of the operators $h_{\bar{f}}^{0, \beta}: H^{2}(\mathbb{D}) \rightarrow \overline{H^{2}(\mathbb{D})}$ and $h_{\bar{f}}^{\beta, 0}: H^{2}(\mathbb{D}) \rightarrow \overline{H^{2}(\mathbb{D})}$ is bounded if and only if $D^{\beta} f \in B M O A$, with $\left\|h_{\bar{f}}^{0, \beta}\right\|_{H^{2}(\mathbb{D}) \rightarrow \overline{H^{2}(\mathbb{D})}}$ and $\left\|h_{\bar{f}}^{\beta, 0}\right\|_{H^{2}(\mathbb{D}) \rightarrow \overline{H^{2}(\mathbb{D})}}$ comparable to $\left\|D^{\beta} f\right\|_{B M O}$.

(ii) Let $\alpha, \beta>0$. Then the operator $h_{\bar{f}}^{\alpha, \beta}: H^{2}(\mathbb{D}) \rightarrow \overline{H^{2}(\mathbb{D})}$ is bounded if and only if $f \in \Lambda_{\alpha+\beta}$, with $\left\|h_{\bar{f}}^{\alpha, \beta}\right\|_{H^{2}(\mathbb{D}) \rightarrow \overline{H^{2}(\mathbb{D})}}$ comparable to $\|f\|_{\Lambda_{\alpha+\beta}}$. 
4.2 Characterizing discrete $\beta$-admissibility of the shift on $H^{2}(\mathbb{D})$.

Similar to the case for the shift on weighted Bergman spaces considered in Section 2, the truth of the weighted Weiss conjecture for the shift on $H^{2}(\mathbb{D})$ is related to whether the operators $h_{\bar{c}}^{\beta, 0}=D^{\beta} h_{\bar{c}}$ and $h_{\bar{c}}^{0, \beta}=h_{\bar{c}} D^{\beta}$ satisfy a RKT. We show that this is true for the former class of operators but false for the latter.

Theorem 4.4. Let $c \in H^{2}(\mathbb{D})$ and $\beta \geq 0$. The following are equivalent:

(i) The operator $h_{\bar{c}}^{\beta, 0}: H^{2}(\mathbb{D}) \rightarrow \overline{H^{2}(\mathbb{D})}$ is bounded.

(ii) The operator $h_{\bar{c}}^{\beta, 0}: H^{2}(\mathbb{D}) \rightarrow \overline{H^{2}(\mathbb{D})}$ is bounded on reproducing kernels, i.e.

$$
M=\sup _{w \in \mathbb{D}}\left(1-|w|^{2}\right)^{1 / 2}\left\|h_{\bar{c}}^{\beta, 0} k_{w}\right\|_{\overline{H^{2}(\mathbb{D})}}<\infty .
$$

Moreover $\left\|h_{\bar{c}}^{\beta, 0}\right\|_{H^{2}(\mathbb{D}) \rightarrow \overline{H^{2}(\mathbb{D})}} \approx M$

Proof. It is obvious that $(i) \Rightarrow(i i)$. By Proposition 4.3 we conclude that $M \lesssim\left\|D^{\beta} c\right\|_{B M O}$ and also that in order to prove $(i i) \Rightarrow(i)$ it is sufficient to show that $\left\|D^{\beta} c\right\|_{B M O} \lesssim \widetilde{M}$. By Proposition 4.1 it is enough to show that

$$
\sum_{J \subseteq I}\left|\left\langle D^{\beta} c, \varphi_{J}\right\rangle\right|^{2} \lesssim M^{2}|I|,
$$

for any dyadic interval $I \subseteq \mathbb{T}$.

Consider a fixed $I$ and define $s_{J}=\left\langle D^{\beta} c, \varphi_{J}\right\rangle$ for $J \subseteq I$. It is immediate that $s_{J}=\left\langle c, D^{\beta} \varphi_{J}\right\rangle$. Since $c$ is analytic,

$$
\begin{aligned}
s_{J} & =\left\langle c, D^{\beta} P \varphi_{J}\right\rangle \\
& =\left\langle c \bar{k}_{w}, h D^{\beta} P \varphi_{J}\right\rangle \\
& =\left\langle P\left(c \bar{k}_{w}\right), h D^{\beta} P \varphi_{J}\right\rangle \\
& =\left\langle g, D^{-\beta}\left(h D^{\beta} P \varphi_{J}\right)\right\rangle,
\end{aligned}
$$

where $w=\sqrt{1-\frac{|I|}{2 \pi}} e^{i x_{I}}, \bar{g}=D^{\beta} h_{\bar{c}} k_{w}$ and $h: \mathbb{D} \rightarrow \mathbb{C}$ is the analytic function given by $h(z)=$ $(1-\bar{w} z)$. Note that $1-|w|^{2}=\frac{|I|}{2 \pi}$ and that by hypothesis $\|g\|^{2} \lesssim \frac{M^{2}}{|I|}$.

It is an elementary exercise to show that

$$
\left(D^{-\beta}\left(h D^{\beta} P \varphi_{J}\right)\right)^{\wedge}(n)=\left(\hat{\varphi}_{J}(n)-\bar{w}\left(\frac{n}{1+n}\right)^{\beta} \hat{\varphi}_{J}(n-1)\right), \quad n \in \mathbb{N}
$$

from which it quickly follows that

$$
D^{-\beta}\left(h D^{\beta} P \varphi_{J}\right)=h \varphi_{J}+\bar{w} F P \varphi_{J},
$$

where

$$
\left(F P \varphi_{J}\right)^{\wedge}(n)=\left(1-\left(\frac{n}{1+n}\right)^{\beta}\right) \hat{\varphi}_{J}(n-1), \quad n \in \mathbb{N}
$$

An important observation is that

$$
\left|1-\left(\frac{x}{1+x}\right)^{\beta}\right| \lesssim \frac{1}{1+x}, \quad x \geq 1
$$


So far, it has been shown that

$$
s_{J}=\left\langle g \bar{h}, P \varphi_{J}\right\rangle+\bar{w}\left\langle g, F P \varphi_{J}\right\rangle
$$

and this expression is now decomposed further. Let $\chi$ be the characteristic function of the interval $\left[x_{I}-2|I|, x_{I}+2|I|\right)$. Then,

$$
\begin{aligned}
s_{J} & =\left\langle g \bar{h}, P \varphi_{J}\right\rangle+\bar{w}\left\langle g, F P \varphi_{J}\right\rangle \\
& =\left\langle\chi g \bar{h}, P \varphi_{J}\right\rangle+\bar{w}\left\langle g, F P \varphi_{J}\right\rangle+\left\langle g,(1-\chi) h P \varphi_{J}\right\rangle,
\end{aligned}
$$

so that

where

$$
\sum_{J \subseteq I}\left|\left\langle D^{\beta} f, \varphi_{J}\right\rangle\right|^{2} \leq 9 \sum_{j=1}^{3} \sum_{J \subset I}\left|s_{J}^{(j)}\right|^{2},
$$

$$
\begin{aligned}
& s_{J}^{(1)}=\left\langle\chi g \bar{h}, P \varphi_{J}\right\rangle, \\
& s_{J}^{(2)}=\left\langle g, F P \varphi_{J}\right\rangle, \\
& s_{J}^{(3)}=\left\langle g,(1-\chi) h P \varphi_{J}\right\rangle .
\end{aligned}
$$

We now handle these three parts separately.

Since $\left\{\varphi_{J}\right\}_{J \subseteq I}$ forms an orthonormal set in $L^{2}(\mathbb{T})$ it follows immediately from Bessel's inequality that

$$
\begin{aligned}
\sum_{J \subseteq I}\left|s_{J}^{(1)}\right|^{2} & \leq\|P \chi \bar{h} g\|^{2} \\
& \leq\|\chi \bar{h} g\|^{2} \\
& =\int_{\left|x-x_{I}\right|<2|I|}\left|g\left(e^{i x}\right)\right|^{2}\left|h\left(e^{-i x}\right)\right|^{2} d x \\
& \leq \sup _{\left|x-x_{I}\right|<2|I|}\left|h\left(e^{-i x}\right)\right|^{2} \frac{M^{2}}{|I|} .
\end{aligned}
$$

Using simple geometric arguments it is easy to show that $\sup _{\left|x-x_{I}\right|<2|I|}\left|h\left(e^{-i x}\right)\right|^{2} \lesssim|I|^{2}$ which in turn gives

$$
\sum_{J \subseteq I}\left|s_{J}^{(1)}\right|^{2} \lesssim M^{2}|I|
$$

The second set of terms is estimated using Hölder's inequality:

$$
\left|s_{J}^{(2)}\right|^{2} \leq\|g\|^{2}\left\|F P \varphi_{J}\right\|^{2} \leq \frac{M^{2}}{|I|}\left\|F P \varphi_{J}\right\|^{2} .
$$

It is easy to show that

$$
\hat{\varphi}_{J}(n)=2^{-j / 2} e^{i n x_{J}} \hat{\psi}\left(\frac{n}{2^{j}}\right) .
$$

Since $\hat{\psi}(\xi)=0$ for $|\xi|<1 / 3$ we have that all $\hat{\varphi}_{J}(n)$ vanish for $n<2^{j} / 3$. Using this together with (14) gives

$$
\begin{aligned}
\left\|F P \varphi_{J}\right\|^{2} & =\sum_{n=0}^{\infty}\left|1-\left(\frac{1+n}{2+n}\right)^{\beta}\right|^{2}\left|\hat{\varphi}_{J}(n)\right|^{2} \\
& \leq\left|1-\left(\frac{1+2^{j} / 3}{2+2^{j} / 3}\right)^{\beta}\right|^{2} \sum_{n=0}^{\infty}\left|\hat{\varphi}_{J}(n)\right|^{2} \\
& \lesssim\left(\frac{1}{2+2^{j} / 3}\right)^{2} \\
& \lesssim|J|^{2} .
\end{aligned}
$$


Hence,

$$
\sum_{J \subseteq I}\left|s_{J}^{(2)}\right|^{2} \lesssim \frac{M^{2}}{|I|} \sum_{J \subseteq I}|J|^{2}=\frac{M^{2}}{|I|} \sum_{n=0}^{\infty} 2^{n}\left(\frac{|I|}{2^{n}}\right)^{2}=2 M^{2}|I|
$$

Estimating the final set of terms is similar to estimating the second, although somewhat more sophisticated. Using Hölder's inequality,

$$
\left|s_{J}^{(3)}\right|^{2} \leq \frac{M^{2}}{|I|}\left\|(1-\chi) h P \varphi_{J}\right\|^{2} .
$$

We now need to show that estimate $\left\|(1-\chi) h \varphi_{J}\right\|^{2} \lesssim|J|^{2}$. To do this, notice that $h=h_{1}+r h_{2}$ where $h_{1}(x)=1-r+r\left(e^{i\left(x-x_{J}\right)}-e^{i\left(x-x_{I}\right)}\right), h_{2}(x)=1-e^{i\left(x-x_{J}\right)}$ and $r=|w|$.

Assuming that $J \subseteq I$ we have that

$$
\left|h_{1}(x)\right| \lesssim(1-r)+\left|x_{J}-x_{I}\right| \lesssim|I|
$$

Now, note that $P \varphi_{J}$ is a periodification of the function $\psi_{+}=\mathcal{F}^{-1}\left(\chi_{\mathbb{R}_{+}} \hat{\psi}\right)$, where $\chi_{\mathbb{R}_{+}}$is the indicator function of $\mathbb{R}_{+}$. This gives

$$
\begin{aligned}
\left|\left(h_{2} P \varphi_{J}\right)(x)\right| & =2^{j / 2}\left|\left(1-e^{i\left(x-x_{J}\right)}\right)\right|\left|\sum_{k \in \mathbb{Z}} \psi_{+}\left(2^{j}\left(x-x_{J}-2 \pi k\right)\right)\right| \\
& \lesssim 2^{j / 2}\left|\left(x-x_{J}\right)\right|\left|\sum_{k \in \mathbb{Z}} \psi_{+}\left(2^{j}\left(x-x_{J}-2 \pi k\right)\right)\right| \\
& \lesssim 2^{j / 2}\left|\sum_{k \in \mathbb{Z}}\left(x-x_{J}-2 \pi k+2 \pi k\right) \psi_{+}\left(2^{j}\left(x-x_{J}-2 \pi k\right)\right)\right| \\
& \leq 2^{-j / 2}\left|\sum_{k \in \mathbb{Z}} 2^{j}\left(x-x_{J}-2 \pi k\right) \psi_{+}\left(2^{j}\left(x-x_{J}-2 \pi k\right)\right)\right| \\
& +2^{j / 2}\left|\sum_{k \in \mathbb{Z}} 2 \pi k \psi_{+}\left(2^{j}\left(x-x_{J}-2 \pi k\right)\right)\right| .
\end{aligned}
$$

Consequently,

$$
\left|\left(h P \varphi_{J}\right)(x)\right| \lesssim \phi_{1}(x)+\phi_{2}(x)+\phi_{3}(x),
$$

where

$$
\begin{aligned}
& \phi_{1}(x)=|I|\left|P \varphi_{J}(x)\right| \\
& \phi_{2}(x)=2^{-j / 2}\left|\sum_{k \in \mathbb{Z}} 2^{j}\left(x-x_{J}-2 \pi k\right) \psi_{+}\left(2^{j}\left(x-x_{J}-2 \pi k\right)\right)\right| \\
& \phi_{3}(x)=2^{j / 2}\left|\sum_{k \in \mathbb{Z}} 2 \pi k \psi_{+}\left(2^{j}\left(x-x_{J}-2 \pi k\right)\right)\right|
\end{aligned}
$$


Now, since $\psi_{+}$is a Schwartz function,

$$
\begin{aligned}
\left\|(1-\chi) \phi_{1}\right\|^{2} & =\int_{2|I|<\left|x-x_{I}\right|<\pi}\left|\phi_{1}(x)\right|^{2} d x \\
& \leq|I|^{2} \int_{|I|<\left|x_{-}\right|<\pi} 2^{j}\left|\sum_{k \in \mathbb{Z}} \psi_{+}\left(2^{j}\left(x-x_{J}-2 \pi k\right)\right)\right|^{2} d x \\
& \lesssim|I|^{2} \int_{|I|<\left|x-x_{J}\right|<\pi} 2^{j} \sum_{k \in \mathbb{Z}}\left|\psi_{+}\left(2^{j}\left(x-x_{J}-2 \pi k\right)\right)\right| d x \\
& \leq|I|^{2} \int_{|I|<\left|x-x_{J}\right|} 2^{j}\left|\psi_{+}\left(2^{j}\left(x-x_{J}\right)\right)\right| d x \\
\left(\operatorname{letting} 2^{j}\left(x-x_{J}\right)=u\right) & =|I|^{2} \int_{2^{j}|I|<|u|}\left|\psi_{+}(u)\right| d x \\
& \lesssim|I|^{2} \int_{2^{j}|I|}^{\infty} \frac{1}{u^{3}} d x \\
& \approx \frac{1}{2^{2 j}} \approx|J|^{2} .
\end{aligned}
$$

In the computation above we have used that $\left|\sum_{k \in \mathbb{Z}} \psi_{+}\left(2^{j}\left(x-x_{J}-2 \pi k\right)\right)\right|$ is uniformly bounded for $x, x_{J} \in \mathbb{R}, j \in \mathbb{N}$.

Similarly $\left\|(1-\chi) \phi_{2}\right\|^{2},\left\|(1-\chi) \phi_{3}\right\|^{2} \lesssim|J|^{2}$. This finally gives

$$
\begin{aligned}
\sum_{J \subseteq I}\left|s_{J}^{(3)}\right|^{2} & \leq \frac{M^{2}}{|I|} \sum_{J \subseteq I}\left\|(1-\chi) h \varphi_{J}\right\|^{2} \\
& \lesssim \frac{M^{2}}{|I|} \sum_{J \subseteq I} \sum_{k=1}^{3}\left\|(1-\chi) \phi_{k}\right\|^{2} \\
& \lesssim \frac{M^{2}}{|I|} \sum_{J \subseteq I}|J|^{2} \\
& =\frac{M^{2}}{|I|} \sum_{n=0}^{\infty} 2^{n}\left(\frac{|I|}{2^{n}}\right)^{2}=2 M^{2}|I|,
\end{aligned}
$$

which completes the proof.

Corollary 4.5. Let $C \in H^{2}(\mathbb{D})^{*}$ and $\beta>0$. Then $C$ is discrete $\beta$-admissible for the shift operator $S: H^{2}(\mathbb{D}) \rightarrow H^{2}(\mathbb{D})$ if and only if

$$
M=\sup _{w \in \mathbb{D}}\left(1-|w|^{2}\right)^{1 / 2}\left\|C(I-\bar{w} S)^{-1}\right\|_{\mathcal{A}_{\beta-1}^{2}(\mathbb{D})^{*}}<\infty .
$$

Moreover $M$ is comparable to the constant of admissibility.

Proof. Let $C f=\langle f, c\rangle$ for $f \in H^{2}(\mathbb{D})$. Then

$$
\begin{aligned}
\left\langle D^{\beta / 2} h_{\bar{c}} k_{w}, \bar{g}\right\rangle & =\left\langle k_{w} D^{\beta / 2} g, c\right\rangle \\
& =\left(1-|w|^{2}\right)^{1 / 2} C(I-\bar{w} S)^{-1} D^{\beta / 2} g,
\end{aligned}
$$

for analytic polynomials $g$. Recalling that $D^{\beta / 2}: H^{2}(\mathbb{D}) \rightarrow \mathcal{A}_{\beta-1}^{2}(\mathbb{D})$ is an isomorphism,

$$
\sup _{w \in \mathbb{D}}\left\|D^{\beta / 2} h_{\bar{c}} k_{w}\right\|_{\overline{H^{2}(\mathbb{D})}}=\sup _{w \in \mathbb{D}}\left(1-|w|^{2}\right)^{1 / 2}\left\|C(I-\bar{w} S)^{-1}\right\|_{\mathcal{A}_{\beta-1}^{2}(\mathbb{D})^{*}} \cdot
$$

The result now follows from Proposition 4.2 and Theorem 4.4. 
4.3 Regarding the failure of $(4) \Rightarrow(3)$.

For $\alpha \in \mathbb{R}$ and $w \in \mathbb{D}$, define the function

$$
g_{w}^{\alpha}(z)=\frac{1}{(1-\bar{w} z)^{1+\alpha}}, \quad z \in \mathbb{D} .
$$

For positive $\alpha, g_{w}^{\alpha}$ is a (non-normalized) reproducing kernel in the Bergman space $\mathcal{A}_{\alpha-1}^{2}(\mathbb{D})$.

Lemma 4.6. Let $\beta>0$ and assume that $c \in \Lambda_{\beta}$. Then for any $\alpha>\beta-1 / 2$ there is a constant $M_{\alpha}$ such that

$$
\sup _{w \in \mathbb{D}}\left(1-|w|^{2}\right)^{1 / 2+\alpha-\beta}\left\|h_{\bar{c}} g_{w}^{\alpha}\right\|_{H^{2}(\mathbb{D})} \leq M_{\alpha}<\infty
$$

Proof. Let $w \in \mathbb{D}$. We will approximate $\left\|h_{\bar{c}} g_{w}^{\alpha}\right\|_{\overline{H^{2}(\mathbb{D})}}$ by $\left\|h_{\bar{c}} g_{w}^{\gamma}\right\|_{\overline{H^{2}(\mathbb{D})}}$ for $\gamma \in(\alpha-\beta, \alpha)$.

Since $c \in \Lambda_{\beta}$, Proposition 4.3 implies that the operator $h_{\bar{c}}^{\alpha-\gamma, \beta-\alpha+\gamma}: H^{2}(\mathbb{D}) \rightarrow \overline{H^{2}(\mathbb{D})}$ is bounded. Using this and boundedness of $D^{-(\beta-\alpha+\gamma)}: \mathcal{A}_{2(\beta-\alpha+\gamma)-1}^{2}(\mathbb{D}) \rightarrow H^{2}(\mathbb{D})$ gives

$$
\begin{aligned}
\left\|h_{\bar{c}} g_{w}^{\gamma}\right\|_{\overline{H^{2}(\mathbb{D})}} & \leq\left\|D^{\alpha-\gamma} h_{\bar{c}} g_{w}^{\gamma}\right\|_{\overline{H^{2}(\mathbb{D})}} \\
& =\left\|D^{\alpha-\gamma} h_{\bar{c}} D^{\beta-\alpha+\gamma} D^{-(\beta-\alpha+\gamma)} g_{w}^{\gamma}\right\|_{\overline{H^{2}(\mathbb{D})}} \\
& \lesssim\left\|D^{-(\beta-\alpha+\gamma)} g_{w}^{\gamma}\right\|_{H^{2}(\mathbb{D})} \\
& =\left\|g_{w}^{\gamma}\right\|_{\mathcal{A}_{2(\beta-\alpha+\gamma)-1}^{2}(\mathbb{D})} .
\end{aligned}
$$

It is well known from the theory of Bergman spaces that

$$
\sup _{w \in \mathbb{D}}\left(1-|w|^{2}\right)^{1 / 2+\alpha-\beta}\left\|g_{w}^{\gamma}\right\|_{\mathcal{A}_{2(\beta-\alpha+\gamma)-1}^{2}(\mathbb{D})}=M_{\alpha}<\infty
$$

for some constant $M_{\alpha}$. Hence, for a fixed $\omega \in \mathbb{D}$,

$$
\left\|h_{\bar{c}} g_{w}^{\gamma}\right\|_{\frac{H^{2}(\mathbb{D})}{}} \leq \frac{M_{\alpha}}{\left(1-|w|^{2}\right)^{1 / 2+\alpha-\beta}} .
$$

In particular $\left\{\left\|h_{\bar{c}} g_{w}^{\gamma}\right\|_{\overline{H^{2}(\mathbb{D})}}\right\}_{\gamma \in(\alpha-\beta, \alpha)}$ is a bounded family of functions, so by reflexivity it has a weakly convergent subsequence as $\gamma \rightarrow \alpha$. Consequently, if it can be shown that

$$
w e a k-\lim _{\gamma \rightarrow \alpha} h_{\bar{c}} g_{w}^{\gamma}=h_{\bar{c}} g_{w}^{\alpha},
$$

then the proof is complete, since then $\left\|h_{\bar{c}} g_{w}^{\alpha}\right\|_{\overline{H^{2}(\mathbb{D})}} \leq \sup _{\gamma \in(\alpha-\beta, \alpha)}\left\|h_{\bar{c}} g_{w}^{\gamma}\right\|_{\overline{H^{2}(\mathbb{D})}}$.

The functions $h_{\bar{c}} g_{w}^{\gamma}$ and $h_{\bar{c}} g_{w}^{\alpha}$ are well defined elements of $H^{2}(\mathbb{D})$ so, for $z \in \mathbb{D}$,

$$
\begin{aligned}
\left(h_{\bar{c}} g_{w}^{\gamma}\right)(z) & =\left\langle\bar{c} g_{w}^{\gamma}, k_{z}\right\rangle \\
& =\int_{\mathbb{T}} \overline{c(\zeta)} \frac{1}{(1-\bar{w} \zeta)^{1+\gamma}} \frac{1}{1-z \bar{\zeta}} d m(\zeta) \\
& \rightarrow \int_{\mathbb{T}} \overline{c(\zeta)} \frac{1}{(1-\bar{w} \zeta)^{1+\alpha}} \frac{1}{1-z \bar{\zeta}} d m(\zeta)=h_{\bar{c}} g_{\omega}^{\alpha}(z), \quad \gamma \rightarrow \alpha
\end{aligned}
$$

where the limit is justified by dominated convergence. This implies that (16) holds.

A consequence of Lemma 4.6 is the following theorem, which is a partial generalization the main result of [22] to $\beta \geq 1$. 
Theorem 4.7. Let $\beta>0$. Then there exists $C_{\beta} \in H^{2}(\mathbb{D})^{*}$ that is not discrete time $\beta$-admissible for $S$, but still satisfies

$$
\sup _{w \in \mathbb{D}}\left(1-|w|^{2}\right)^{\frac{1-\beta}{2}+\alpha}\left\|C_{\beta}(I-\bar{w} S)^{-(1+\alpha)}\right\|_{H^{2}(\mathbb{D})^{*}}<\infty
$$

for any $\alpha>\frac{\beta-1}{2}$.

Proof. The space $D^{-\beta / 2} B M O A$ is strictly included in $\Lambda_{\beta / 2}^{+}$. Choose $c_{\beta} \in \Lambda_{\beta / 2}^{+} \backslash D^{-\beta / 2} B M O A$ and define $C_{\beta}$ by

$$
C_{\beta} f=\left\langle f, c_{\beta}\right\rangle, \quad f \in H^{2}(\mathbb{D}) .
$$

By Theorem 4.3, this operator is not $\beta$-admissible for $S$. However, it satisfies the resolvent condition by Lemma 4.6 .

We remark that Lemma 4.6 still holds if $g_{w}^{\alpha}$ is replaced by $D^{\alpha} k_{w}$. The proof is preserved, word for word, except that the standard Bergman space estimate (15) is replaced by the estimate

$$
\left\|D^{\alpha-\beta} k_{w}\right\|_{H^{2}(\mathbb{D})} \lesssim \frac{1}{\left(1-|w|^{2}\right)^{1 / 2+\alpha-\beta}}
$$

This in turn follows from the estimate

$$
\frac{\prod_{i=1}^{n}(i+\gamma)}{n !}=\frac{n^{\gamma}}{\Gamma(1+\gamma)}\left\{1+O\left(\frac{1}{n}\right)\right\}, \quad n \in \mathbb{N}, \gamma \notin\{-1,-2, \ldots\},
$$

see [25, Chap. 3]. This modified version of Lemma 4.6 immediately implies the following counterexample.

Theorem 4.8. Let $\beta>0$. Then there exists $c \in H^{2}(\mathbb{D})$ such that the generalized Hankel operator $h_{\bar{c}}^{0, \beta}: H^{2}(\mathbb{D}) \rightarrow \overline{H^{2}(\mathbb{D})}$ does not satisfy the reproducing kernel thesis.

\section{Admissibility of the right-shift semigroup on $L^{2}\left(\mathbb{R}_{+}\right)$}

We begin with a number of technical definitions which are required in order to characterise $\beta$ admissibility, $\beta>0$, of the right-shift semigroup on $L^{2}\left(\mathbb{R}_{+}\right)$.

5.1 Distribution spaces and the $\varphi$-transform.

Let $\check{\phi}$ denote the inverse Fourier transform $\mathcal{F}^{-1} \phi$ and define the space

$$
\mathcal{Z}=\left\{\phi \in \mathcal{S}: \check{\phi}^{(k)}=0, k=0,1,2, \ldots\right\}
$$

with the topology inherited from the Schwartz space $\mathcal{S}$. Its topological dual $\mathcal{Z}^{\prime}$ is isomorphic to $\mathcal{S}^{\prime} / \mathcal{P}$ (the space of tempered distributions modulo polynomials). Much of the notation used in this section is taken from [2]. For a slightly more detailed introduction to the space $\mathcal{Z}^{\prime}$ we refer to $[16]$.

Let $\varphi \in \mathcal{S}$ be a function such that

$$
\begin{gathered}
\operatorname{supp} \hat{\varphi} \subseteq\{\xi \in \mathbb{R}: 1 / 2 \leq|\xi| \leq 2\} \\
|\hat{\varphi}(\xi)| \geq c>0 \quad \text { for } 3 / 5 \leq|\xi| \leq 5 / 3,
\end{gathered}
$$


and also

$$
\sum_{n=-\infty}^{\infty} \hat{\varphi}\left(2^{-n} \xi\right)=1, \quad \xi \in \mathbb{R} \backslash\{0\}
$$

For each $n \in \mathbb{Z}$ define

$$
\varphi_{n}(x)=2^{n} \varphi\left(2^{n} x\right), \quad x \in \mathbb{R} .
$$

From [3, Lemma 6.9] we cite the following lemma.

Lemma 5.1. Assume that $\varphi$ satisfies (17) and (18). Then there exists $\psi \in \mathcal{S}$ that also satisfies (17) and (18) and that

$$
\sum_{n \in \mathbb{Z}} \overline{\hat{\varphi}\left(2^{n} \xi\right)} \hat{\psi}\left(2^{n} \xi\right)=1, \quad \xi \neq 0
$$

We remark that in [2] the existence of $\psi$ as in the above lemma was stated as a requirement in the choice of $\varphi$.

Let $\alpha \in \mathbb{R}$ and $0<p, q \leq \infty$. The Besov space $\dot{B}_{p}^{\alpha, q}$ is defined as the set of $f \in \mathcal{Z}^{\prime}$ such that

$$
\|f\|_{\dot{B}_{p}^{\alpha, q}}:=\left\|\left\{2^{n \alpha}\left\|\varphi_{n} * f\right\|_{L^{p}}\right\}_{n \in \mathbb{Z}}\right\|_{l^{q}(\mathbb{Z})}<\infty .
$$

If $p<\infty$, define the Triebel-Lizorkin space $\dot{F}_{p}^{\alpha, q}$ as the set of $f \in \mathcal{Z}^{\prime}$ such that

$$
\|f\|_{\dot{F}_{p}^{\alpha, q}}:=\left\|\left(\sum_{n \in \mathbb{Z}}\left(2^{n \alpha}\left|\varphi_{n} * f\right|\right)^{q}\right)^{1 / q}\right\|_{L^{p}}<\infty .
$$

The exceptional space $\dot{F}_{\infty}^{\alpha, q}$ is defined as the set of $f \in \mathcal{Z}^{\prime}$ such that

$$
\|f\|_{\dot{F}_{\infty}^{\alpha, q}}:=\sup \left(\frac{1}{|P|} \int_{P} \sum_{n=-\log _{2}|P|}^{\infty}\left(2^{n \alpha}\left|\varphi_{n} * f\right|\right)^{q}\right)^{1 / q}<\infty,
$$

where the supremum is taken over all dyadic intervals $P=\left[k 2^{-j},(k+1) 2^{-j}\right)$. The last definition is the one given in [2] where it is also proved to be equivalent to the definition given in [16]. The definitions can be proved to be independent of the choice of $\varphi,[16$, p. 240].

Given a function $g: \mathbb{R} \rightarrow \mathbb{C}$ we define the multiplication operator $M_{g(x)}: \mathcal{Z}^{\prime} \rightarrow \mathcal{Z}^{\prime}$ by

$$
\left(M_{g(x)} f\right)(x)=g(x) f(x), \quad f \in \mathcal{Z}^{\prime},
$$

provided that this is well defined. For $\alpha \in \mathbb{R}$, define the inverse Riesz potential $D^{\alpha}: \mathcal{Z}^{\prime} \rightarrow \mathcal{Z}^{\prime}$ by

$$
D^{\alpha} f=\mathcal{F}\left(M_{|\xi|^{\alpha}} \check{f}\right) .
$$

From [16, p. 242, Theorem 1; p. 244, Theorem] we collect the following results.

Proposition 5.2. Let $\alpha, \beta \in \mathbb{R}$ and $0<p, q \leq \infty$. Then $D^{\beta}: \dot{B}_{p}^{\alpha, q} \rightarrow \dot{B}_{p}^{\alpha-\beta, q}$ is a surjective isomorphism. If $p<\infty$, then $D^{\beta}: \dot{F}_{p}^{\alpha, q} \rightarrow \dot{F}_{p}^{\alpha-\beta, q}$ is a surjective isomorphism. Moreover we have the special cases $\dot{F}_{2}^{0,2}=L^{2}$ and $\dot{F}_{\infty}^{0,2}=B M O(\mathbb{R})$.

We remark that by the duality identity $\left(F_{1}^{\alpha, q}\right)^{*}=F_{\infty}^{-\alpha, q^{\prime}}$ (see [2, Equation (5.2)]) valid for $q \in[1, \infty)$ the conclusion of Proposition 5.2 holds also for the spaces $\dot{F}_{\infty}^{\alpha, q}$ whenever $q \in(1, \infty]$. This shows in particular that $D^{-\alpha} B M O(\mathbb{R})=\dot{F}_{\infty}^{\alpha, 2}$.

Given $\phi \in \mathcal{Z}$, define $P_{+} \phi=\mathcal{F}\left(\chi_{\mathbb{R}_{+}} \check{\phi}\right)$ where $\chi_{\mathbb{R}_{+}}$denotes the indicator function of the positive real numbers. Also define $P_{+}: \mathcal{Z}^{\prime} \rightarrow \mathcal{Z}^{\prime}$ by

$$
\left\langle\phi, P_{+} f\right\rangle=\left\langle P_{+} \phi, f\right\rangle, \quad \phi \in \mathcal{Z}, f \in \mathcal{Z}^{\prime},
$$


and $P_{-}=I-P_{+}$. Test functions and distributions belonging to $\mathcal{Z}_{+}=P_{+} \mathcal{Z}$ and $\mathcal{Z}_{+}^{\prime}=P_{+} \mathcal{Z}^{\prime}$ respectively will be referred to as analytic. The main reason for this is that

$$
P_{+} L^{2}=H^{2}\left(\mathbb{C}_{+}\right),
$$

the Hardy space of the right half plane.

Let $\alpha \in \mathbb{R}$ and $0<p, q \leq \infty$. We will work with sequences $s=\left\{s_{Q}\right\}_{Q}$ indexed by the set of dyadic intervals on $\mathbb{R}$. If $p<\infty$, define the space $\dot{f}_{p}^{\alpha, q}$ of sequences such that

$$
\|s\|_{\dot{f}_{p}^{\alpha, q}}:=\left\|\left(\sum_{Q}\left(|Q|^{-\alpha}\left|s_{Q}\right| \tilde{\chi}_{Q}\right)^{q}\right)^{1 / q}\right\|_{L^{p}}<\infty
$$

where $\tilde{\chi}_{Q}=|Q|^{-1 / 2} \chi_{Q}$ is the $L^{2}$-normalized indicator function of $Q$. In the special case $p=q=2$ the integral in the above norm is easily computed and we have that

$$
\|s\|_{\dot{f}_{2}^{\alpha, 2}}=\left(\sum_{Q}|Q|^{-2 \alpha}\left|s_{Q}\right|^{2}\right)^{1 / 2} .
$$

The space $\dot{f}_{\infty}^{\alpha, q}$ is defined by the norm

$$
\|s\|_{\dot{f}_{\infty}^{\alpha, q}}:=\sup _{P \text { dyadic }}\left(\frac{1}{|P|} \int_{P} \sum_{Q \subseteq P}\left(|Q|^{-\alpha}\left|s_{Q}\right| \tilde{\chi}_{Q}\right)^{q}\right)^{1 / q} .
$$

In particular we have that

$$
\|s\|_{\dot{f}_{\infty}^{\alpha, 2}}=\sup _{P \text { dyadic }}\left(\frac{1}{|P|} \sum_{Q \subseteq P}|Q|^{-2 \alpha}\left|s_{Q}\right|^{2}\right)^{1 / 2}
$$

For each dyadic interval $Q$, define the dilation translation

$$
\varphi_{Q}(x)=|Q|^{-1 / 2} \varphi\left(\frac{x-x_{Q}}{|Q|}\right), \quad x \in \mathbb{R},
$$

where $x_{Q}$ is the left endpoint of $Q$. The sequence $\left\{\psi_{Q}\right\}_{Q}$ is defined similarly. We define the $\varphi$-transform of a distribution $f \in \mathcal{Z}^{\prime}$ by

$$
S_{\varphi} f=\left\{\left\langle f, \varphi_{Q}\right\rangle\right\}_{Q}
$$

The inverse $\varphi$-transform of a sequence $s=\left\{s_{Q}\right\}_{Q}$ is defined by

$$
T_{\psi} s=\sum_{Q} s_{Q} \psi_{Q}
$$

where $\psi$ is as in Lemma 5.1. The importance of the $\varphi$-transform is the following ([2, Theorem $2.2])$.

Proposition 5.3. Let $\alpha \in \mathbb{R}$ and $0<p, q \leq \infty$. The operators $S_{\varphi}: \dot{F}_{p}^{\alpha, q} \rightarrow \dot{f}_{p}^{\alpha, q}$ and $T_{\psi}: \dot{f}_{p}^{\alpha, q} \rightarrow \dot{F}_{p}^{\alpha, q}$ are bounded. Moreover $T_{\psi} \circ S_{\phi}$ is the identity on $\dot{F}_{p}^{\alpha, q}$. In particular $\|f\|_{\dot{F}_{p}^{\alpha, q}} \approx\left\|S_{\phi} f\right\|_{\dot{f}_{p}^{\alpha, q}}$. 
5.2 Continuous time admissibility and Hankel operators.

Let $c \in \mathcal{Z}_{-}^{\prime}$. Define the Hankel type operator $H_{c}: \mathcal{Z}_{+} \rightarrow \mathcal{Z}_{-}^{\prime}$ by

$$
H_{c} f=P_{-}(c f), \quad f \in \mathcal{Z}_{+} .
$$

First we observe that the operator $H_{c}$ can be defined in a natural way on a larger class of functions that just $\mathcal{Z}_{+}$. Let $f \in \mathcal{Z}_{+}^{\prime}$. Formally,

$$
\begin{aligned}
\left\langle\phi, H_{c} f\right\rangle & =\langle\phi, c f\rangle \\
& =\langle\check{\phi}, \check{c} * \check{f}\rangle \\
& =\langle\langle\check{\phi}(\xi+\eta), \check{f}(\eta)\rangle, \check{c}(\xi)\rangle, \quad \phi \in \mathcal{Z}_{-} .
\end{aligned}
$$

The last expression is well defined provided that $\langle\check{\phi}(\xi+\eta), \check{f}(\eta)\rangle$ is a Schwartz function that vanishes on $\mathbb{R}_{+}$. This is the case if, for example, $f=K_{\lambda}$ where

$$
K_{\lambda}(x)=\frac{1}{\bar{\lambda}+i x}, \quad x \in \mathbb{R}
$$

for some $\lambda \in \mathbb{C}_{+}$, since then

$$
\check{K}_{\lambda}(\xi)= \begin{cases}e^{-\xi \bar{\lambda}} & \text { if } \xi \geq 0, \\ 0 & \text { if } \xi<0 .\end{cases}
$$

If $K_{\lambda}$ is thought of as a function of $i x$ rather than of $x$, then $K_{\lambda}$ is a reproducing kernel of $H^{2}\left(\mathbb{C}_{+}\right)$ with respect to $\lambda$. We therefore say that the operator $H_{c}$ is defined on the set of reproducing kernels. For future convenience we also define $K_{\lambda}^{\gamma}=D^{\gamma} K_{\lambda}$ whenever $\gamma \in \mathbb{R}$. If $\gamma>0$ then $K_{\lambda}^{\gamma}$ (multiplied with a constant) is a reproducing kernel for the space $\mathcal{A}_{\gamma-1}^{2}\left(\mathbb{C}_{+}\right)$. As long as $\gamma>-1$ we have that the map $H_{c} K_{\lambda}^{\gamma}: \mathcal{Z}_{-} \rightarrow \mathbb{C}$ is well defined.

Let $(S(t))_{t \geq 0}$ be the right shift semigroup on $L^{2}\left(\mathbb{R}_{+}\right)$. Its infinitesimal generator $A$ is given by

$$
A=-\frac{d}{d t}, \quad D(A)=W_{0}^{1,2}\left(\mathbb{R}_{+}\right)=\left\{f \in L^{2}\left(\mathbb{R}_{+}\right): f^{\prime} \in L^{2}\left(\mathbb{R}_{+}\right), f(0)=0\right\}
$$

see [17, Example 2.4.5] for details. If $C \in D(A)^{*}$ then $C(I-A)^{-1} \in L^{2}\left(\mathbb{R}_{+}\right)^{*}$ and so there is a unique $c_{0} \in L^{2}\left(\mathbb{R}_{+}\right)$such that

$$
C(I-A)^{-1} f=\left\langle f, c_{0}\right\rangle, \quad f \in L^{2}\left(\mathbb{R}_{+}\right) .
$$

Conversely this equation generates an $A$-bounded linear functional for any $c_{0} \in L^{2}\left(\mathbb{R}_{+}\right)$.

In order to compute fractional power resolvents, the following lemma ([5, Proposition 3.3.5]) is useful.

Lemma 5.4. Let $B$ be the generator of the contractive $C_{0}$-semigroup $(T(t))_{t \geq 0}$ on a Banach space $X$. Then for any $\lambda \in \mathbb{C}_{+}$and $\beta>0$

$$
(\lambda I-B)^{-\beta}=\frac{1}{\Gamma(\beta)} \int_{0}^{\infty} t^{\beta-1} T(t) e^{-t \lambda} d t .
$$

A simple consequence is the following.

Lemma 5.5. Let $f \in \mathcal{Z}_{+}, \lambda \in \mathbb{C}_{+}, \beta>0$ and $A$ be the infinitesimal generator of the right-shift semigroup on $L^{2}\left(\mathbb{R}_{+}\right)$. Then

$$
\mathcal{F}\left((\bar{\lambda} I-A)^{-(1+\beta)} \check{f}\right)=f K_{\lambda}^{\beta} .
$$

The following proposition relates reproducing kernels and generalised Hankel operators to the weighted Weiss conjecture. 
Proposition 5.6. Let $A$ be the infinitesimal generator of the right shift semigroup, $C \in D(A)^{*}$ and $\beta \geq 0$. If $c_{0} \in L^{2}\left(\mathbb{R}_{+}\right)$is related to $C$ through (21) and $c \in \mathcal{Z}_{-}^{\prime}$ is given by

$$
c(\xi)=(1+i \xi)\left(\mathcal{F} \tilde{c}_{0}\right)(\xi), \quad \xi \in \mathbb{R}
$$

where $\tilde{c}_{0}(s)=\overline{c_{0}(-s)}$. Then:

(i) Whenever $f \in \mathcal{Z}_{+}$,

$$
\int_{0}^{\infty} t^{2 \beta}|C S(t) \check{f}|^{2} d t=\left\|D^{\beta} H_{c} f\right\|_{L^{2}(\mathbb{R})}^{2} .
$$

In particular, since $\mathcal{F}^{-1} \mathcal{Z}_{+}$is dense in $D(A), C$ is $2 \beta$-admissible for $(S(t))_{t \geq 0}$ if and only if (ii) $D^{\beta} H_{c}: \mathcal{Z}_{+} \rightarrow L^{2}(\mathbb{R})$ extends to a bounded linear operator from $\mathcal{F} L^{2}\left(\mathbb{R}_{+}\right)$to $L^{2}(\mathbb{R})$.

$$
\left\|C(\bar{\lambda} I-A)^{-1} M_{|x|^{\beta}}\right\|_{L^{2}\left(\mathbb{R}_{+}, d x\right)^{*}}=\left\|D^{\beta} H_{C} K_{\lambda}\right\|_{L^{2}(\mathbb{R})} .
$$

In particular the resolvent estimate

$$
\sup _{\lambda \in \mathbb{C}_{+}}(\operatorname{Re} \lambda)^{1 / 2}\left\|C(\bar{\lambda} I-A)^{-1} M_{|x|^{\beta}}\right\|_{L^{2}\left(\mathbb{R}_{+}, d x\right)^{*}}<\infty
$$

(iii)

holds if and only if $D^{\beta} H_{c}: \mathcal{Z}_{+} \rightarrow L^{2}(\mathbb{R})$ is bounded on reproducing kernels.

$$
\left\|C(\bar{\lambda} I-A)^{-(1+\beta)}\right\|_{L^{2}\left(\mathbb{R}_{+}, d x\right)^{*}}=\left\|H_{C} K_{\lambda}^{\beta}\right\|_{L^{2}(\mathbb{R})} .
$$

In particular the resolvent estimate

$$
\sup _{\lambda \in \mathbb{C}_{+}}(\operatorname{Re} \lambda)^{1 / 2}\left\|C(\bar{\lambda} I-A)^{-(1+\beta)}\right\|_{L^{2}\left(\mathbb{R}_{+}, d x\right)^{*}}<\infty
$$

holds if and only if $H_{c} D^{\beta}: \mathcal{Z}_{+} \rightarrow L^{2}(\mathbb{R})$ is bounded on reproducing kernels.

Proof. (i) Take $f \in \mathcal{Z}_{+}$and consider the function

$$
g_{f}: t \mapsto \begin{cases}C S(-t) \check{f} & \text { if } t \leq 0 \\ 0 & \text { if } t>0\end{cases}
$$

Then, for $t \leq 0$,

$$
\begin{aligned}
g_{f}(t) & =C(I-A)^{-1}(I-A) S(-t) \check{f} \\
& =\left\langle(I-A) S(-t) \check{f}, c_{0}\right\rangle \\
& =\int_{s=-t}\left(\check{f}+(\check{f})^{\prime}\right)(s+t) \overline{c_{0}(s)} d s \\
& =\left(\check{f}+(\check{f})^{\prime}\right) * \tilde{c}_{0}(t) .
\end{aligned}
$$

Hence,

$$
\begin{aligned}
& \int_{t=0}^{\infty} t^{2 \beta}|C S(t) \check{f}|^{2} d t=\int_{t=-\infty}^{0}|t|^{2 \beta}|C S(-t) \check{f}|^{2} d t \\
& =\left\|M_{|t|^{\beta}} \chi_{\mathbb{R}_{-}}\left(\left(\check{f}+(\check{f})^{\prime}\right) * \tilde{c}_{0}\right)\right\|_{L^{2}}^{2} \\
& =\left\|D^{\beta} P_{-}\left((1+i \xi) f \hat{\tilde{c}}_{0}\right)\right\|_{L^{2}} \\
& =\left\|D^{\beta} H_{c} f\right\|_{L^{2}} \text {. }
\end{aligned}
$$


(ii) Using Lemma 5.5 together with the elementary identity $\bar{f}=\mathcal{F} \tilde{\tilde{f}}$ we obtain,

$$
\begin{aligned}
C(\bar{\lambda} I-A)^{-1} M_{|x|^{\beta}} \check{f} & =C(I-A)^{-1}(I-A)(\bar{\lambda} I-A)^{-1} M_{|x|^{\beta}} \check{f} \\
& =\left\langle(I-A)(\bar{\lambda} I-A)^{-1} M_{|x|^{\beta}} \check{f}, c_{0}\right\rangle \\
& =\left\langle(1+i \xi) K_{\lambda} D^{\beta} f, \hat{c}_{0}\right\rangle \\
& =\left\langle(1+i \xi) K_{\lambda} \overline{\hat{c}}_{0}, D^{\beta} \bar{f}\right\rangle \\
& =\left\langle D^{\beta} H_{c} K_{\lambda}, \hat{\tilde{\tilde{f}}}\right\rangle .
\end{aligned}
$$

The result follows by taking the supremum over all $f \in \mathcal{Z}_{+}$of unit length.

(iii) This is similar to the proof of $(i i)$.

In the paper [9] the authors characterize boundedness of the operators $D^{\alpha} H_{c} D^{\beta}$ for $\alpha, \beta \geq$ 0. In particular we need [9, Theorem 5.1] and [9, Theorem 5.3] (together with a few technical comments from the examples).

Proposition 5.7. For $c \in \mathcal{Z}_{-}^{\prime}$ we have that:

(i) Let $\beta \geq 0$. Then both $D^{\beta} H_{c}: \mathcal{Z}_{+} \rightarrow L^{2}(\mathbb{R})$ and $H_{c} D^{\beta}: \mathcal{Z}_{+} \rightarrow L^{2}(\mathbb{R})$ extend to bounded linear operators from $\mathcal{F} L^{2}\left(\mathbb{R}_{+}\right)$to $L^{2}(\mathbb{R})$ if and only if $D^{\beta} c \in B M O(\mathbb{R})$, with $\left\|D^{\beta} H_{c}\right\|_{\mathcal{F} L^{2}\left(\mathbb{R}_{+}\right) \rightarrow L^{2}(\mathbb{R})}$ and $\left\|H_{c} D^{\beta}\right\|_{\mathcal{F} L^{2}\left(\mathbb{R}_{+}\right) \rightarrow L^{2}(\mathbb{R})}$ comparable to $\left\|D^{\beta} c\right\|_{B M O(\mathbb{R})}$.

(ii) Let $\alpha, \beta>0$. Then $D^{\alpha} H_{c} D^{\beta}: \mathcal{Z}_{+} \rightarrow L^{2}(\mathbb{R})$ extends to a bounded linear operator from $\mathcal{F} L^{2}\left(\mathbb{R}_{+}\right)$ to $L^{2}(\mathbb{R})$ if and only if $c \in \dot{B}_{\infty}^{\alpha+\beta}$, with $\left\|D^{\alpha} H_{c} D^{\beta}\right\|_{\mathcal{F} L^{2}\left(\mathbb{R}_{+}\right) \rightarrow L^{2}(\mathbb{R})}$ comparable to $\|c\|_{\dot{B}_{\infty}^{\alpha+\beta}}$.

5.3 The Reproducing Kernel Thesis for $D^{\alpha} H_{c}$.

The main result of this section is the following.

Theorem 5.8. Let $c \in \mathcal{Z}_{-}^{\prime}$ and $\beta \geq 0$. The following are equivalent:

(i) The operator $D^{\beta} H_{c}: \mathcal{Z}_{+} \rightarrow L^{2}(\mathbb{R})$ extends to a bounded operator on $H^{2}\left(\mathbb{C}_{+}\right)$;

(ii) The operator $D^{\beta} H_{c}: \mathcal{Z}_{+} \rightarrow L^{2}(\mathbb{R})$ is bounded on reproducing kernels, i.e.

$$
M=\sup _{\lambda \in \mathbb{C}_{+}}(\operatorname{Re} \lambda)^{1 / 2}\left\|D^{\beta} H_{c} K_{\lambda}\right\|_{L^{2}(\mathbb{R})}<\infty .
$$

Moreover $\left\|D^{\beta} H_{c}\right\|_{H^{2}\left(\mathbb{C}_{+}\right) \rightarrow L^{2}(\mathbb{R})} \approx M$.

Proof. $(i) \Rightarrow($ ii $)$ is trivial. By Proposition 5.7 the converse statement follows if $\left\|D^{\beta} c\right\|_{B M O(\mathbb{R})} \lesssim M$. By Proposition 5.2 we need to show that $c \in \dot{F}_{\infty}^{\beta, 2}$ which by Proposition 5.3 is equivalent to that $S_{\varphi} c \in \dot{f}_{\infty}^{\beta, 2}$. Define the sequence $s_{Q}=\left\langle c, \varphi_{Q}\right\rangle$. It is sufficient to prove that

$$
\sup _{P \text { dyadic }}\left(\frac{1}{|P|} \sum_{Q \subseteq P}|Q|^{-2 \beta}\left|s_{Q}\right|^{2}\right)^{1 / 2} \lesssim M .
$$

To this end, consider a fixed dyadic interval $P$. Since $c \in \mathcal{Z}_{-}^{\prime}$,

$$
s_{Q}=\left\langle c K_{\lambda}, \bar{h} P_{-} \varphi_{Q}\right\rangle=\left\langle H_{c} K_{\lambda}, \bar{h} P_{-} \varphi_{Q}\right\rangle=\left\langle g, D^{-\beta}\left(\bar{h} P_{-} \varphi_{Q}\right)\right\rangle,
$$


where $\lambda=|P|+i x_{P}, g=D^{\beta} H_{c} K_{\lambda}$ and $h(x)=\bar{\lambda}+i x$. In the above calculation we have used that $\bar{h} P_{-} \varphi_{Q} \in \mathcal{Z}_{-}$. Note that

$$
\|g\|_{L^{2}(\mathbb{R})}^{2} \leq \frac{M^{2}}{|P|}
$$

Let $\chi \in \mathcal{S}$ be a smooth cutoff such that $\chi(x)=1$ when $\left|x-x_{P}\right| \leq 2|P|, 0<\chi(x)<1$ when $2|P|<\left|x-x_{P}\right|<3|P|$ and $\chi(x)=0$ when $\left|x-x_{P}\right| \geq 3|P|$. We have

$$
\begin{aligned}
s_{Q}=\left\langle g, D^{-\beta}\left(\bar{h} P_{-} \varphi_{Q}\right)\right\rangle & =\left\langle\chi g+(1-\chi) g, D^{-\beta}\left(\bar{h} P_{-} \varphi_{Q}\right)\right\rangle \\
& =\left\langle h D^{-\beta}(\chi g), P_{-} \varphi_{Q}\right\rangle+\left\langle g,(1-\chi) D^{-\beta}\left(h P_{-} \varphi_{Q}\right)\right\rangle .
\end{aligned}
$$

A calculation shows that

$$
\begin{aligned}
h D^{-\beta}(\chi g) & =\mathcal{F}\left(\left[\bar{\lambda}+\frac{d}{d \xi}\right] M_{|\xi|-\beta}(\check{\chi} * \check{g})\right) \\
& =\mathcal{F}\left(\bar{\lambda} M_{|\xi|-\beta}(\check{\chi} * \check{g})\right)+\mathcal{F}\left(M_{|\xi|-\beta} \frac{d}{d \xi}(\check{\chi} * \check{g})\right)-\mathcal{F}\left(\beta M_{\operatorname{sgn}(\xi)|\xi|^{-(1+\beta)}}(\check{\chi} * \check{g})\right) \\
& =D^{-\beta}(h \chi g)-\beta D^{-(1+\beta)} \mathcal{F}\left(M_{\operatorname{sgn}(\xi)}(\check{\chi} * \check{g})\right) .
\end{aligned}
$$

This implies that $s_{Q}=s_{Q}^{(1)}+s_{Q}^{(2)}+s_{Q}^{(3)}$, where

$$
\begin{aligned}
& s_{Q}^{(1)}=\left\langle D^{-\beta}(h \chi g), P_{-} \varphi_{Q}\right\rangle, \\
& s_{Q}^{(2)}=-\beta\left\langle D^{-(1+\beta)} \mathcal{F}\left(M_{\operatorname{sgn}(\xi)}(\check{\chi} * \check{g})\right), P_{-} \varphi_{Q}\right\rangle, \\
& s_{Q}^{(3)}=\left\langle g,(1-\chi) D^{-\beta}\left(\bar{h} P_{-} \varphi_{Q}\right)\right\rangle .
\end{aligned}
$$

The proof is completed by showing that

$$
\sum_{Q \subseteq P}|Q|^{-2 \beta}\left|s_{Q}^{(j)}\right|^{2} \lesssim M^{2}|P|, \quad j=1,2,3
$$

First, using Propositions 5.2 and 5.3,

$$
\begin{aligned}
\sum_{Q \subseteq P}|Q|^{-2 \beta}\left|s_{Q}^{(1)}\right|^{2} & =\sum_{Q \subseteq P}|Q|^{-2 \beta}\left|\left\langle D^{-\beta} P_{-}(h \chi g), \varphi_{Q}\right\rangle\right|^{2} \\
& \leq \sum_{Q}|Q|^{-2 \beta}\left|\left\langle D^{-\beta} P_{-}(h \chi g), \varphi_{Q}\right\rangle\right|^{2} \\
& =\left\|S_{\varphi}\left(D^{-\beta} P_{-}(h \chi g)\right)\right\|_{\dot{f}_{2}^{\beta, 2}} \\
& \lesssim\left\|D^{-\beta} P_{-}(h \chi g)\right\|_{\dot{F}_{2}^{\beta, 2}} \\
& \approx\left\|P_{-}(h \chi g)\right\|_{\dot{F}_{2}^{0,2}} \\
& \approx\left\|P_{-}(h \chi g)\right\|_{L^{2}} \\
& \leq \int_{\left|x-x_{P}\right|<3|P|}|\bar{\lambda}+i x|^{2}|g(x)|^{2} d x \lesssim|P| M^{2}
\end{aligned}
$$


The second part is estimated using Hölders inequality on each term.

$$
\begin{aligned}
\left|s_{Q}^{(2)}\right|^{2} & =\beta^{2}\left|\left\langle D^{-(1+\beta)} \mathcal{F}\left(M_{\operatorname{sgn}(\xi)}(\check{\chi} * \check{g})\right), P_{-} \varphi_{Q}\right\rangle\right|^{2} \\
& =\beta^{2}\left|\left\langle M_{\operatorname{sgn}(\xi)|\xi|^{-(1+\beta)}}(\check{\chi} * \check{g}), \chi_{\mathbb{R}_{-}} \check{\varphi}_{Q}\right\rangle\right|^{2} \\
& \leq \beta^{2}\left(\int_{-\frac{2}{|Q|}}^{-\frac{1}{2|Q|}}|\xi|^{-(1+\beta)}|(\check{\chi} * \check{g})(\xi)|\left|\check{\varphi}_{Q}(\xi)\right| d \xi\right)^{2} \\
& \lesssim|Q|^{2+2 \beta}\|\check{\chi} * \check{g}\|_{L^{2}(\mathbb{R})}^{2} \\
& \leq|Q|^{2+2 \beta}\|g\|_{L^{2}(\mathbb{R})}^{2} \\
& \leq \frac{|Q|^{2+2 \beta} M^{2}}{|P|} .
\end{aligned}
$$

In the calculation we have used that $\left\{\varphi_{Q}\right\}_{Q}$ is an $L^{2}$-normalized sequence. Summing up,

$$
\sum_{Q \subseteq P}|Q|^{-2 \beta}\left|s_{Q}^{(2)}\right|^{2} \leq \sum_{Q \subseteq P} \frac{|Q|^{2} M^{2}}{|P|}=\sum_{n=0}^{\infty} 2^{n}\left(\frac{|P|}{2^{n}}\right)^{2} \frac{M^{2}}{|P|}=2 M^{2}|P| .
$$

Finally, apply Hölders inequality to each term in the third and final sum to give

$$
\left|s_{Q}^{(3)}\right|^{2} \leq \frac{M^{2}}{|P|}\left\|(1-\chi) D^{-\beta}\left(\bar{h} P_{-} \varphi_{Q}\right)\right\|_{L^{2}}^{2} .
$$

Before summing up we need to approximate $\left\|(1-\chi) D^{-\beta}\left(\bar{h} P_{-} \varphi_{Q}\right)\right\|_{L^{2}}^{2}$. It is elementary to show that

$$
\check{\varphi}_{Q}(\xi)=|Q|^{1 / 2} e^{i x_{Q} \xi} \check{\varphi}(|Q| \xi), \quad \xi \in \mathbb{R} .
$$

This gives that for $x \in \mathbb{R}$,

$$
\begin{aligned}
D^{-\beta}\left(\bar{h} P_{-} \varphi_{Q}\right)(x) & =\mathcal{F}\left(M _ { | \xi | } [ \lambda - \frac { d } { d \xi } ] \left(\chi_{\left.\left.\mathbb{R}_{-} \check{\varphi}_{Q}\right)\right)(x)}\right.\right. \\
& =\int_{-\infty}^{0}|\xi|^{-\beta}|Q|^{1 / 2} e^{i x_{Q} \xi}\left[\left(\lambda-i x_{Q}\right) \check{\varphi}(|Q| \xi)-|Q|(\check{\varphi})^{\prime}(|Q| \xi)\right] e^{-i \xi x} d \xi \\
(\operatorname{letting} u=|Q| \xi) & =|Q|^{\beta-1 / 2} \int_{-\infty}^{0}|u|^{-\beta}\left[\left(\lambda-i x_{Q}\right) \check{\varphi}(u)+|Q|(\check{\varphi})^{\prime}(u)\right] e^{-i u\left(\frac{x-x_{Q}}{|Q|}\right)} d u \\
& =|Q|^{\beta-1 / 2}\left[\left(\lambda-i x_{Q}\right) D^{-\beta} P_{-} \varphi+|Q| D^{-\beta}\left(M_{i x} P_{-} \varphi\right)\right]\left(\frac{x-x_{Q}}{|Q|}\right) .
\end{aligned}
$$

Assuming $Q \subseteq P$ we then obtain

$$
\left|D^{-\beta}\left(\bar{h} P_{-} \varphi_{Q}\right)(x)\right|^{2} \lesssim|P|^{2}|Q|^{2 \beta-1}\left|\phi\left(\frac{x-x_{Q}}{|Q|}\right)\right|, \quad x \in \mathbb{R},
$$

where

Using (23),

$$
\phi=\left|D^{-\beta} P_{-} \varphi\right|^{2}+\left|D^{-\beta}\left(M_{i x} P_{-} \varphi\right)\right|^{2} .
$$

$$
\begin{aligned}
\left\|(1-\chi) D^{-\beta}\left(\bar{h} P-\varphi_{Q}\right)\right\|_{L^{2}}^{2} & \leq \int_{\left|x-x_{P}\right|>2|P|}\left|D^{-\beta}\left(\bar{h} P-\varphi_{Q}\right)(x)\right|^{2} d x \\
& \leq \int_{\left|x-x_{Q}\right|>|P|}|P|^{2}|Q|^{2 \beta-1}\left|\phi\left(\frac{x-x_{Q}}{|Q|}\right)\right| d x \\
\left(\text { letting } u=\frac{x-x_{Q}}{|Q|}\right) & =\int_{|u|>\frac{|P|}{|Q|}}|P|^{2}|Q|^{2 \beta}|\phi(u)| d u .
\end{aligned}
$$


Since $\phi$ decays like a Schwartz function,

$$
|\phi(x)| \lesssim \frac{1}{|x|^{3}}, \quad x \in \mathbb{R}
$$

and hence,

$$
\begin{aligned}
\left\|(1-\chi) D^{-\beta}\left(\bar{h} \varphi_{Q}\right)\right\|_{L^{2}}^{2} & \lesssim \int_{|x|>\frac{|P|}{|Q|}}|P|^{2}|Q|^{2 \beta} \frac{1}{|x|^{3}} d x \\
& \lesssim|Q|^{2+2 \beta} .
\end{aligned}
$$

Combining the above inequality with (22) gives

$$
\left|s_{Q}^{(3)}\right|^{2} \lesssim \frac{M^{2}}{|P|}|Q|^{2+2 \beta}
$$

Summing up,

$$
\sum_{Q \subseteq P}|Q|^{-2 \beta}\left|s_{Q}^{(3)}\right|^{2} \lesssim \sum_{Q \subseteq P} \frac{M^{2}}{|P|}|Q|^{2}=\sum_{n=0}^{\infty} \frac{M^{2}}{|P|} 2^{n}\left(\frac{|P|}{2^{n}}\right)^{2}=2 M^{2}|P|
$$

Corollary 5.9. Let $A$ denote the infinitesimal generator of the right shift semigroup $(S(t))_{t \geq 0}$ and $\beta \geq 0$. Then $C \in D(A)^{*}$ is $2 \beta$-admissible for $(S(t))_{t \geq 0}$ if and only if

$$
M=\sup _{\lambda \in \mathbb{C}_{+}}(\operatorname{Re} \lambda)^{1 / 2}\left\|C(\bar{\lambda} I-A)^{-1} M_{|x|^{\beta}}\right\|_{L^{2}\left(\mathbb{R}_{+}, d x\right)^{*}}<\infty .
$$

Moreover the constant $M$ is comparable to the constant of admissibility.

Proof. This follows immediately from Theorem 5.8 and Proposition 5.6.

5.4 Regarding the failure of $(2) \Rightarrow(1)$.

We have the following analogue of Lemma 4.6.

Lemma 5.10. Let $\beta>0$ and $c \in \mathcal{Z}_{-}^{\prime}$. Then the following statements are true:

(i) If for some $\alpha>\beta-1 / 2$ there exists a constant $M_{\alpha}$ such that

$$
M_{\alpha}=\sup _{\lambda \in \mathbb{C}_{+}}(\operatorname{Re} \lambda)^{1 / 2+\alpha-\beta}\left\|H_{c} K_{\lambda}^{\alpha}\right\|_{H^{2}\left(\mathbb{C}_{+}\right)}<\infty
$$

then $c \in \dot{B}_{\infty}^{\beta}$.

(ii) If $\alpha>\max \{\beta-1 / 2,0\}, c \in \dot{B}_{\infty}^{\beta}$ and moreover

$$
\frac{c(x)}{1+i x} \in L^{2}(\mathbb{R})
$$

then there exists a constant $M_{\alpha}$ such that

$$
M_{\alpha}=\sup _{\lambda \in \mathbb{C}_{+}}(\operatorname{Re} \lambda)^{1 / 2+\alpha-\beta}\left\|H_{c} K_{\lambda}^{\alpha}\right\|_{H^{2}\left(\mathbb{C}_{+}\right)}<\infty .
$$


Proof. In order to prove the first statement let $\left\{\varphi_{n}\right\}_{n \in \mathbb{Z}}$ be as in section 5.1. We need to show that

$$
\left|\left\langle g, \varphi_{n} * c\right\rangle\right| \lesssim 2^{-n \beta}\|g\|_{L^{1}}, \quad n \in \mathbb{Z}, g \in \mathcal{Z} .
$$

Since $c \in \mathcal{Z}_{-}^{\prime}$ we may replace $\varphi_{n}$ with $P_{-} \varphi_{n}$.

Consider fixed $n \in \mathbb{Z}, g \in \mathcal{Z}$. By definition of the convolution,

$$
\left\langle g, \varphi_{n} * c\right\rangle=\langle\phi, c\rangle
$$

where $\phi: x \mapsto\left\langle g(x+y), \varphi_{n}(y)\right\rangle$.

Introduce the functions $g_{k}=g \chi_{\left[k 2^{-n},(k+1) 2^{-n}\right)}$ and $\phi_{k}: x \mapsto\left\langle g_{k}(x+y), \varphi_{n}(y)\right\rangle$, where $\chi_{\left[k 2^{-n},(k+1) 2^{-n}\right)}$ denotes the characteristic function of $\left[k 2^{-n},(k+1) 2^{-n}\right)$. A quick calculation shows that $\hat{\phi}_{k}=\hat{g}_{k} \overline{\hat{\varphi}_{n}}$ so that $\phi_{k} \in \mathcal{Z}_{-}$. It is also easy to show that $\phi=\sum_{k \in \mathbb{Z}} \phi_{k}$ with convergence in the Schwartz topology.

Choose the sequence $\lambda_{k}=2^{-n}+i k 2^{-n}, k \in \mathbb{Z}$. Then

$$
\begin{aligned}
\left\langle g, \varphi_{n} * c\right\rangle & =\sum_{k \in \mathbb{Z}}\left\langle\phi_{k}, c\right\rangle \\
& =\sum_{k \in \mathbb{Z}}\left\langle\phi_{k}(x)\left(\lambda_{k}-i x\right)^{1+\alpha}, c(x) D^{\alpha} K_{\lambda_{k}}(x)\right\rangle \\
& =\sum_{k \in \mathbb{Z}}\left\langle\phi_{k}(x)\left(\lambda_{k}-i x\right)^{1+\alpha},\left(H_{c} D^{\alpha} K_{\lambda_{k}}\right)(x)\right\rangle .
\end{aligned}
$$

This gives

$$
\begin{aligned}
\left|\left\langle g, \varphi_{n} * c\right\rangle\right| & \leq \sum_{k \in \mathbb{Z}}\left|\left\langle\phi_{k}(x)\left(\lambda_{k}-i x\right)^{1+\alpha},\left(H_{c} D^{\alpha} K_{\lambda_{k}}\right)(x)\right\rangle\right| \\
& \leq \sum_{k \in \mathbb{Z}}\left\|\phi_{k}(x)\left(\lambda_{k}-i x\right)^{1+\alpha}\right\|_{L^{2}}\left\|\left(H_{c} D^{\alpha} K_{\lambda_{k}}\right)(x)\right\|_{L^{2}} \\
& \leq \frac{M_{\alpha}}{2^{-n(1 / 2+\alpha-\beta)}} \sum_{k \in \mathbb{Z}}\left\|\phi_{k}(x)\left(\lambda_{k}-i x\right)^{1+\alpha}\right\|_{L^{2}} .
\end{aligned}
$$

We come down to approximating $\left\|\phi_{k}(x)\left(\lambda_{k}-i x\right)^{1+\alpha}\right\|_{L^{2}}$. Applying Minkowski's inequality in the first step gives

$$
\begin{aligned}
\left\|\phi_{k}(x)\left(\lambda_{k}-i x\right)^{1+\alpha}\right\|_{L^{2}} & \leq \int\left|g_{k}(y)\right|\left\|\varphi_{n}(y-x)\left(\lambda_{k}-i x\right)^{1+\alpha}\right\|_{L^{2}(d x)} d y \\
& =\left\|g_{k}\right\|_{L^{1}} \sup _{y \in\left[k 2^{-n},(k+1) 2^{-n}\right)}\left\|\varphi_{n}(x)\left(\lambda_{k}-i(y-x)\right)^{1+\alpha}\right\|_{L^{2}(d x)^{2}} .
\end{aligned}
$$

By a change of variables, it follows that for each $y \in\left[k 2^{-n},(k+1) 2^{-n}\right)$,

$$
\begin{aligned}
\left\|\varphi_{n}(x)\left(\bar{\lambda}_{k}-i(y-x)\right)^{1+\alpha}\right\|_{L^{2}(d x)}^{2} \lesssim & 2^{n}\left(\left|2^{-n}+i\left(y-k 2^{-n}\right)\right|^{2+2 \alpha} \int\left|\varphi_{0}(x)\right|^{2} d x\right. \\
& \left.+2^{-2 n(1+\alpha)} \int|x|^{2+2 \alpha}\left|\varphi_{0}(x)\right|^{2} d x\right) \\
\lesssim & 2^{-n(1+2 \alpha)} .
\end{aligned}
$$

Consequently,

$$
\begin{aligned}
\left|\left\langle g, \varphi_{n} * c\right\rangle\right| & \leq \frac{M_{\alpha}}{2^{-n(1 / 2+\alpha-\beta)}} \sum_{k \in \mathbb{Z}}\left\|\phi_{k}(x)\left(\lambda_{k}+i x\right)^{1+\alpha}\right\|_{L^{2}} \\
& \lesssim \frac{M_{\alpha}}{2^{n \beta}} \sum_{k \in \mathbb{Z}}\left\|g_{k}\right\|_{L^{1}}=M_{\alpha} 2^{-n \beta}\|g\|_{L^{1}},
\end{aligned}
$$


and hence, $c \in \dot{B}_{\infty}^{\beta}$.

To prove the second statement, fix $\lambda \in \mathbb{C}_{+}$, let $\gamma \in(\max \{\alpha-\beta, 0\}, \alpha)$ and consider the functions $H_{c} K_{\lambda}^{\gamma}$. Note that

$$
\begin{aligned}
\left\|H_{c} K_{\lambda}^{\gamma}\right\| & \leq\left\|\chi_{(-1,0)} M_{|\xi|^{\alpha-\gamma}}\left(\check{c} * \check{K}_{\lambda}^{\gamma}\right)\right\|+\left\|\chi_{(-1,0)} M_{1-|\xi|^{\alpha-\gamma}}\left(\check{c} * \check{K}_{\lambda}^{\gamma}\right)\right\|+\left\|\chi_{(-\infty,-1)} M_{|\xi|^{\alpha-\gamma}}\left(\check{c} * \check{K}_{\lambda}^{\gamma}\right)\right\| \\
& \lesssim\left\|\chi_{(-1,0)} M_{|\xi|^{\alpha-\gamma}}\left(\check{c} * \check{K}_{\lambda}^{\gamma}\right)\right\|+\left\|D^{\alpha-\gamma} H_{c} D^{\beta-\alpha+\gamma} K_{\lambda}^{\alpha-\beta}\right\| .
\end{aligned}
$$

By the assumption $(24), H_{c} K_{\lambda}^{\gamma} \rightarrow H_{c} K_{\lambda}^{\alpha}$ with convergence in $L^{2}(\mathbb{R})$ as $\gamma \rightarrow \alpha$. Hence, it is easy

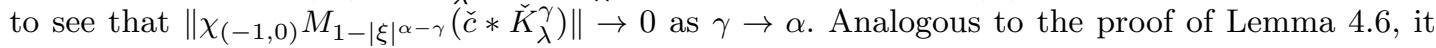
follows that

$$
\left\|D^{\alpha-\gamma} H_{c} D^{\beta-\alpha+\gamma} K_{\lambda}^{\alpha-\beta}\right\| \lesssim\left\|K_{\lambda}^{\alpha-\beta}\right\| \approx(\operatorname{Re} \lambda)^{-1 / 2+\beta-\alpha},
$$

which completes the proof.

Lemma 5.10 will provide a counterexample to the weighted Weiss conjecture once it has been proven that a certain set of operator symbols is nonempty.

Lemma 5.11. Let $\beta>0$. Then there exists $c \in \mathcal{Z}_{-}^{\prime}$ with the following properties:

(i)

(ii)

$$
\frac{c(x)}{1+i x} \in L^{2}(\mathbb{R})
$$

$$
D^{\beta} c \notin B M O(\mathbb{R})
$$

(iii)

$$
D^{\beta} c \in \dot{B}_{\infty}^{0}
$$

Proof. We construct $c$ explicitly. Let $f_{0} \in \mathcal{S}$ be a smooth function with $\operatorname{supp}\left(\check{f}_{0}\right) \subset[-1,-1 / 2]$ and let $f=M_{1+i x} f_{0}$. Define $c \in \mathcal{Z}_{-}^{\prime}$ by

$$
\check{c}(\xi)=\sum_{n=0}^{\infty} \frac{\check{f}\left(\xi+2^{n}\right)}{|\xi|^{\beta}}, \quad \xi \in \mathbb{R} .
$$

It is clear that $c \in L^{2}(\mathbb{R})$ and consequently $(i)$ holds.

Furthermore $D^{\beta} c=M_{1+i x} g$ where

$$
\check{g}(\xi)=\sum_{n=0}^{\infty} \check{f}_{0}\left(\xi+2^{n}\right), \quad \xi \in \mathbb{R} .
$$

Since clearly $g \notin L^{2}(\mathbb{R})$ we have that $D^{\beta} c \notin B M O(\mathbb{R})$.

Finally, since the support of $\check{c}$ is sparse,

$$
\left|\varphi_{n} *\left(D^{\beta} c\right)(x)\right|=\left|\int \varphi_{n}(x-y) f(y)\left(e^{i 2^{n} y}+e^{i 2^{n+1} y}\right) d y\right| \leq 2\|f\|_{L^{\infty}(\mathbb{R})}\left\|\varphi_{n}\right\|_{L^{1}(\mathbb{R})},
$$

which by the normalization of $\left\{\varphi_{n}\right\}_{n \in \mathbb{Z}}$ is uniformly bounded for $x \in \mathbb{R}, n \in \mathbb{Z}$.

Theorem 5.12. Let $\beta>0$ and let $A$ be the infinitesimal generator of the right shift semigroup on $L^{2}\left(\mathbb{R}_{+}\right)$. There exists an operator $C_{\beta} \in D(A)^{*}$ which is not $2 \beta$-admissible but still satisfies

$$
\sup _{\lambda \in \mathbb{C}_{+}}(\operatorname{Re} \lambda)^{1 / 2+\alpha-\beta}\left\|C(\bar{\lambda} I-A)^{-(1+\alpha)}\right\|_{L^{2}\left(\mathbb{R}_{+}\right)^{*}}<\infty
$$

for any $\alpha>\max \{\beta-1 / 2,0\}$. 
Proof. Let $c_{\beta} \in \mathcal{Z}_{-}^{\prime}$ have the properties stated in Lemma 5.11. By the first property there is a corresponding observation operator $C_{\beta} \in D(A)^{*}$. By Lemma 5.10 this operator satisfies the resolvent condition while by Propositions 5.6 and 5.7 it is not $2 \beta$-admissible.

Analogous to the discrete time case, we obtain the following result.

Theorem 5.13. Let $\beta>0$. There exists $c \in \mathcal{Z}_{-}^{\prime}$ such that the operator $H_{c} D^{\beta}: H^{2}\left(\mathbb{C}_{+}\right) \rightarrow L^{2}(\mathbb{R})$ does not satisfy the reproducing kernel thesis.

\section{References}

1. P. Duren, E. A. Gallardo-Gutiérrez, and A. Montes-Rodríguez. A Paley-Wiener theorem for Bergman spaces with application to invariant subspaces. Bull. Lond. Math. Soc., 39(3):459-466, 2007.

2. M. Frazier and B. Jawerth. A discrete transform and decompositions of distribution spaces. J. Funct. Anal., 93(1):34-170, 1990.

3. M. Frazier, B. Jawerth, and G. Weiss. Littlewood-Paley theory and the study of function spaces, volume 79 of CBMS Regional Conference Series in Mathematics. Published for the Conference Board of the Mathematical Sciences, Washington, DC, 1991.

4. B. Haak and C. Le Merdy. $\alpha$-admissibility of observation and control operators. Houston J. Math., 31(4):1153-1167, 2005.

5. M. Haase. The functional calculus for sectorial operators, volume 169 of Operator Theory: Advances and Applications. Birkhäuser Verlag, Basel, 2006.

6. Z. Harper. Applications of the discrete Weiss conjecture in operator theory. Integral Equations Operator Theory, 54(1):69-88, 2006.

7. B. Jacob and J. R. Partington. The Weiss conjecture on admissibility of observation operators for contraction semigroups. Integral Equations Operator Theory, 40(2):231-243, 2001.

8. S. Janson. Hankel operators between weighted Bergman spaces. Ark. Mat., 26(2):205-219, 1988.

9. S. Janson and J. Peetre. Paracommutators-boundedness and Schatten-von Neumann properties. Trans. Amer. Math. Soc., 305(2):467-504, 1988.

10. Y. Meyer. Wavelets and operators, volume 37 of Cambridge Studies in Advanced Mathematics. Cambridge University Press, Cambridge, 1992.

11. N. K. Nikolski. Operators, functions, and systems: an easy reading. Vol. 1, volume 92 of Mathematical Surveys and Monographs. American Mathematical Society, Providence, RI, 2002.

12. J. R. Partington and G. Weiss. Admissible observation operators for the right-shift semigroup. Math. Control Signals Systems, 13(3):179-192, 2000.

13. V. V. Peller. Vectorial Hankel operators, commutators and related operators of the Schatten-von Neumann class $\gamma_{p}$. Integral Equations Operator Theory, 5(2):244-272, 1982.

14. V. V. Peller. Hankel operators and their applications. Springer Monographs in Mathematics. SpringerVerlag, New York, 2003.

15. B. Sz.-Nagy, C. Foias, H. Bercovici, and L. Kérchy. Harmonic analysis of operators on Hilbert space. Universitext. Springer, New York, second edition, 2010.

16. H. Triebel. Theory of function spaces. Modern Birkhäuser Classics. Birkhäuser/Springer Basel AG, Basel, 2010

17. M. Tucsnak and G. Weiss. Observation and control for operator semigroups. Birkhäuser Advanced Texts: Basler Lehrbücher. Birkhäuser Verlag, Basel, 2009.

18. G. Weiss. Two conjectures on the admissibility of control operators. In Estimation and control of distributed parameter systems (Vorau, 1990), volume 100 of Internat. Ser. Numer. Math., pages 367-378. Birkhäuser, Basel, 1991.

19. G. Weiss. A powerful generalization of the Carleson measure theorem? In Open problems in mathematical systems and control theory, Comm. Control Engrg. Ser., pages 267-272. Springer, London, 1999.

20. G. Weiss, O. J. Staffans, and M. Tucsnak. Well-posed linear systems - a survey with emphasis on conservative systems. Int. J. Appl. Math. Comput. Sci., 11(1):7-33, 2001. Mathematical theory of networks and systems (Perpignan, 2000).

21. A. Wynn. $\alpha$-admissibility of the right-shift semigroup on $L^{2}\left(\mathbb{R}_{+}\right)$. Systems Control Lett., 58(9):677-681, 2009.

22. A. Wynn. Counterexamples to the discrete and continuous weighted Weiss conjectures. SIAM J. Control Optim., 48(4):2620-2635, 2009.

23. A. Wynn. $\alpha$-admissibility of observation operators in discrete and continuous time. Complex Anal. Oper. Theory, 4(1):109-131, 2010.

24. K. Zhu. Operator theory in function spaces, volume 138 of Mathematical Surveys and Monographs. American Mathematical Society, Providence, RI, second edition, 2007.

25. A. Zygmund. Trigonometric series. 2nd ed. Vol. I. Cambridge University Press, New York, 1959. 